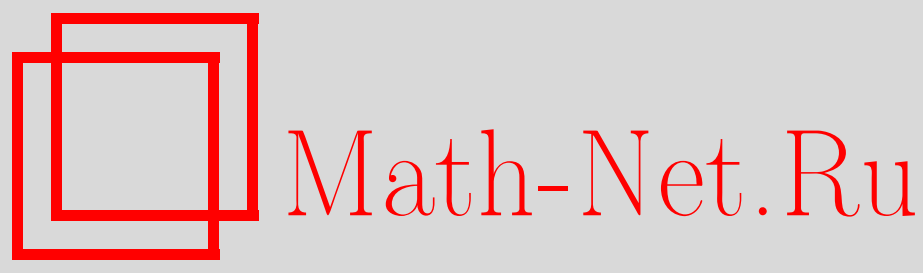

А. Ю. Аникин, Квантовые нормальные формы Биркгофа, ТMФ, 2009, том 160, номер 3, 487-506

DOI: https://doi.org/10.4213/tmf6411

Использование Общероссийского математического портала Math-Net.Ru подразумевает, что вы прочитали и согласны с пользовательским соглашением http: //www.mathnet.ru/rus/agreement

Параметры загрузки:

IP : 44.207 .124 .84

26 апреля 2023 г., 13:58:11

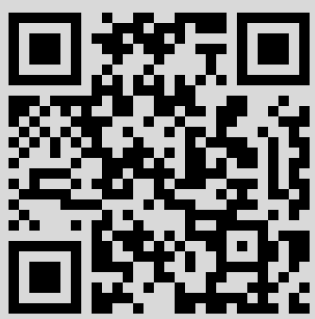




\section{КВАНТОВЫЕ НОРМАЛЬНЫЕ ФОРМЫ БИРКГОФА}

Рассмотрены квантовые аналоги ряда теорем о нормальных формах Биркгофа. Доказаны квантовый аналог теоремы о приведении гамильтониана к нормальной форме и аналог теоремы о приведении гамильтониана к вещественной нормальной форме. Получен явный вид нормальной формы в нерезонансном случае. Рассматриваются вопросы единственности нормальной формы и нормализующего преобразования в нерезонансном и резонансном случаях.

Ключевые слова: нормальная форма Биркгофа, квантовый аналог, алгебра наблюдаемых, автоморфизм.

\section{1. ВВЕДЕНИЕ}

В классической механике нормальные формы представляют собой мощный инструмент локального анализа динамических систем. Под нормальной формой понимают простейший вид, к которому можно привести систему дифференциальных уравнений заменой переменных в окрестности особенности (положения равновесия, периодического решения или инвариантного тора). Этой теории посвящены работы известных авторов: Пуанкаре, Дюлака, Биркгофа, Черри, Мозера, Зигеля, Брюно и многих других [1]-[4].

Нормальные формы для гамильтоновых систем впервые были построены Биркгофом и Черри [1], [2], они называются нормальными формами Биркгофа. В этих работах изучается, к какому простейшему виду можно привести гамильтониан системы в окрестности положения равновесия с помощью канонического преобразования. Теория нормальных форм Биркгофа является формальной, так как в ней гамильтониан и нормализующее преобразование представляют собой степенные ряды, о сходимости которых ничего не говорится.

Наша задача состоит в том, чтобы построить аналог нормальных форм Биркгофа для квантовых наблюдаемых. В духе работ [5], [6] мы рассматриваем квантовую механику с нетрадиционных позиций. Под квантовыми наблюдаемыми мы будем

* Московский государственный технический университет им. Н.Э. Баумана, Москва, Россия. E-mail: anikin83@inbox.ru 
понимать элементы некоммутативной ассоциативной алгебры формальных рядов. Также множество наблюдаемых имеет структуру алгебры Ли относительно операции коммутатора. Под каноническими преобразованиями понимаются автоморфизмы этой алгебры. Интерпретация этих алгебраических объектов как операторов в $L^{2}\left(\mathbb{R}^{n}\right)$ в настоящей работе не рассматривается.

Отметим, что идея нормальных форм в квантовой механике не нова. С помощью техники интегрального оператора Фурье [7]-[9] в работах [10], [11] строятся квантовые нормальные формы вблизи устойчивого положения равновесия, а в работах [12]-[14] - вблизи неустойчивого. Более общие результаты получены в работе [15].

При традиционном подходе для квантования классических объектов используют символьные отображения. Такие отображения определены не единственным образом и не являются гомоморфизмами алгебр классических и квантовых наблюдаемых. Наиболее распространенное отображение - символ Вейля. Используемый нами альтернативный подход выглядит более естественным для исследования нормальных форм. Кроме того, он позволяет получать не только формальные теоремы, но и в некоторых случаях доказывать сходимость. В работе [16] доказана аналитичность нормализующего преобразования для квантового аналога теоремы Мозера о нормальной форме гамильтоновой системы с полутора степенями свободы.

Из других работ по квантовым нормальным формам, использующим традиционный подход, отметим работу [17], где нормальные формы строятся с помощью метода квантового усреднения [18]. Эта работа ближе по духу к нашей, чем указанные выше. В ней строятся нормальные формы, а также исследуются алгебры Ли, порожденные нормальными формами. Эти алгебры Ли нетривиальны для резонансного случая. Некоторые простые резонансы разобраны в работе [18] достаточно подробно.

Методы и техника, используемые нами, аналогичны тем, которые используются в классической механике. Разница состоит лишь в том, что в классическом случае мы имеем дело с коммутативными объектами, а в квантовом - с некоммутативными. Из наших результатов о нормальных формах классические результаты получаются как тривиальные следствия после применения специального гомоморфизма квантовой и классической алгебр.

Сформулируем наиболее важный результат настоящей работы - теорему о приведении квантовой наблюдаемой к нормальной форме. Сначала дадим алгебраическую формулировку классического результата Биркгофа. Рассмотрим ассоциативную алгебру $\mathbb{O}_{n}$ над $\mathbb{C}$ коммутативных формальных степенных рядов со стандартным произведением

$$
F=\sum_{\mu, \nu \in \mathbb{Z}_{+}^{n}} f_{\mu, \nu} p_{1}^{\mu_{1}} \ldots p_{n}^{\mu_{n}} x_{1}^{\nu_{1}} \ldots x_{n}^{\nu_{n}}=\sum_{\mu, \nu \in \mathbb{Z}_{+}^{n}} f_{\mu, \nu} p^{\mu} x^{\nu}, \quad f_{\mu, \nu} \in \mathbb{C}
$$

где $\mu=\left(\mu_{1}, \ldots, \mu_{n}\right), \nu=\left(\nu_{1}, \ldots, \nu_{n}\right)$. Образующие $p_{1}, \ldots, p_{n}, x_{1}, \ldots, x_{n}$ этой коммутативной алгебры можно интерпретировать как классические координаты и импульсы механической системы в фазовом пространстве $\mathbb{R}^{2 n}$. Нижний индекс в обозначении алгебры $\mathbb{O}_{n}$ мы, как правило, будем опускать. 
Ассоциативная алгебра (O) также имеет структуру алгебры Ли по отношению к стандартной скобке Пуассона:

$$
\{F, G\}=\sum_{j=1}^{n} \frac{\partial F}{\partial p_{j}} \frac{\partial G}{\partial x_{j}}-\frac{\partial F}{\partial x_{j}} \frac{\partial G}{\partial p_{j}} .
$$

Элементы алгебры $\mathbb{O}$ называются классическими наблюдаемыми.

Биективные линейные отображения $\mathcal{A}: \mathbb{O} \rightarrow \mathbb{O}$ называются автоморфизмами ассоциативной алгебры и алгебры Ли $\mathbb{O}$, если

$$
\mathcal{A}(F G)=\mathcal{A} F \mathcal{A} G, \quad \mathcal{A}\{F, G\}=\{\mathcal{A} F, \mathcal{A} G\} .
$$

В классической механике такие отображения называются каноническими преобразованиями.

Сформулируем теорему Биркгофа о приведении к нормальной форме.

Рассмотрим классическую наблюдаемую $H=H_{2}+H_{3}+H_{4}+\cdots \in \mathbb{O}$, где $H_{j}-$ однородная форма степени $j$, причем $H_{2}=\sum_{k=1}^{n} \lambda_{k} p_{k} x_{k}$. Тогда существует такое отображение $\mathcal{A} \in$ Aut $\mathbb{O}$, что $\mathcal{A} H=H_{2}+\mathcal{H}_{3}+\mathcal{H}_{4}+\cdots$, причем $\left\{\mathcal{A} H, H_{2}\right\}=0$.

Для любых $l, \lambda \in \mathbb{Z}^{n}$ обозначим через $\langle l, \lambda\rangle$ стандартное скалярное произведение. Назовем моном $f_{\mu, \nu} p^{\mu} x^{\nu}$ резонансным, если $\langle k, \mu-\nu\rangle=0$ для всех ненулевых $k \in \mathbb{Z}^{n}$. Несложно увидеть, что соотношение $\left\{\mathcal{A} H, H_{2}\right\}=0$ эквивалентно тому, что в разложении нормальной формы $\mathcal{A} H$ имеются лишь резонансные мономы.

Сформулируем аналогичную квантовую теорему. Сначала построим свободную некоммутативную ассоциативную алгебру формальных рядов с образующими $\hat{p}_{j}, \hat{x}_{j}$ и произведением о. Определим мономы следующим образом:

$$
\hat{z}=\hat{z}_{1} \circ \ldots \circ \hat{z}_{k}, \quad \hat{z}_{1}, \ldots, \hat{z}_{k} \in\left\{\hat{x}_{1}, \ldots, \hat{x}_{n}, \hat{p}_{1}, \ldots, \hat{p}_{n}\right\}
$$

Число $k$ называется степенью монома, $\operatorname{deg} \hat{z}=k$. Подчеркнем, что мономы равны, если они построены из одних и тех же элементов, взятых в одинаковом порядке.

Рассмотрим следующее линейное пространство над $\mathbb{C}$ некоммутативных формальных рядов:

$$
\widehat{\mathbb{O}}_{n}=\left\{\widehat{F}=\sum_{k=0}^{\infty} \sum_{\operatorname{deg} \hat{z}=k} f_{\hat{z}} \hat{z}, \quad f_{\hat{z}} \in \mathbb{C}\right\},
$$

где суммирование проводится по всем мономам $\hat{z}$.

В $\widehat{\mathbb{O}}_{n}$ определено ассоциативное произведение о. Таким образом, $\left(\widehat{\mathbb{O}}_{n},+, \circ\right)$ является свободной ассоциативной алгеброй. Нижний индекс в обозначении $\widehat{\mathbb{O}}_{n}$ мы в дальнейшем также будем, как правило, опускать.

Положим $\mathbf{r}_{j}:=\hat{p}_{j} \circ \hat{x}_{j}-\hat{x}_{j} \circ \hat{p}_{j}$ и рассмотрим алгебру $\widehat{\mathbb{H}}$, порожденную следующими тождествами:

$$
\begin{gathered}
\hat{p}_{j} \circ \hat{p}_{s}-\hat{p}_{s} \circ \hat{p}_{j}=0, \quad \hat{x}_{j} \circ \hat{x}_{s}-\hat{x}_{s} \circ \hat{x}_{j}=0, \\
\mathbf{r}_{j}-\mathbf{r}_{s}=0, \quad \mathbf{r}_{j} \circ \hat{p}_{s}-\hat{p}_{s} \circ \mathbf{r}_{j}=0, \quad \mathbf{r}_{j} \circ \hat{x}_{s}-\hat{x}_{s} \circ \mathbf{r}_{j}=0
\end{gathered}
$$

для всех $s, j=1, \ldots, n$, а также элементами

$$
\hat{p}_{j} \circ \hat{x}_{s}-\hat{x}_{s} \circ \hat{p}_{j}=0, \quad s, j=1, \ldots, n, \quad s \neq j .
$$


А именно, мы рассматриваем фактор-алгебру $\widehat{\mathbb{H}}=\widehat{\mathbb{O}} / \hat{J}$, где $\hat{J}$ есть идеал, порожденный левыми частями тождеств $(3)-(5)$. Обозначим через $\pi: \widehat{\mathbb{O}} \rightarrow \widehat{\mathbb{H}}$ гомоморфизм проекции. Введем обозначение $\mathbf{r}=\pi \mathbf{r}_{j}$.

Ассоциативная алгебра $\widehat{\mathbb{H}}$ называется алгеброй квантовых наблюдаемых. В стандартном представлении образующие $\hat{x}_{j}$ и $\hat{p}_{j}$ отождествляются со следующими операторами в $L_{2}\left(\mathbb{R}^{n}\right)$ :

$$
\hat{x}_{j} f(x)=x_{j} f(x), \quad \hat{p}_{j} f(x)=-i \hbar \frac{\partial f}{\partial x_{j}}(x) .
$$

Элемент $\mathbf{r}$ отвечает константе $-i \hbar$ (точнее, оператору $-i \hbar \mathrm{id})$.

Коммутатор вводит структуру алгебры Ли на $\widehat{\mathbb{H}}$. Точное определение коммутатора мы дадим ниже, неформально он определяется следующим образом:

$$
[\widehat{F}, \widehat{G}]=\frac{\widehat{F} \circ \widehat{G}-\widehat{G} \circ \widehat{F}}{\mathbf{r}}, \quad \widehat{F}, \widehat{G} \in \widehat{\mathbb{H}} .
$$

Биективные линейные отображения $\widehat{\mathcal{A}}: \widehat{\mathbb{H}} \rightarrow \widehat{\mathbb{H}}$, сохраняющие операции о и $[\cdot, \cdot]$, назовем автоморфизмами ассоциативной алгебры и алгебры Ли $\widehat{\mathbb{H}}$. Группа таких автоморфизмов обозначается Aut $\widehat{\mathbb{H}}$.

Существует гомоморфизм aver: $\widehat{\mathbb{H}} \rightarrow \mathbb{O}$ ассоциативных алгебр и алгебр Ли

$$
\operatorname{aver}(\widehat{F} \circ \widehat{G})=\operatorname{aver} \widehat{F} \circ \text { aver } \widehat{G}, \quad \text { aver }[\widehat{F}, \widehat{G}]=[\text { aver } \widehat{F} \text {, aver } \widehat{G}]
$$

называемый усреднением. Он получается отождествлением $\mathbf{r}=0$. Благодаря действию этого гомоморфизма все классические результаты являются тривиальными следствиями своих квантовых аналогов.

Сформулируем один из наших основных результатов - квантовый аналог теоремы Биркгофа.

Рассмотрим квантовую наблюдаемую $\widehat{H}=\widehat{H}_{2}+\widehat{H}_{3}+\widehat{H}_{4}+\cdots \in \widehat{\mathbb{H}}$, где $\widehat{H}_{j}-$ однородные формы степени $j$, причем $\widehat{H}_{2}=\sum_{k=1}^{n} \lambda_{k} \hat{p}_{k} \circ \hat{x}_{k}$. Тогда существует такое отображение $\widehat{\mathcal{A}} \in$ Aut $\widehat{\mathbb{H}}$, что $\widehat{\mathcal{A}} \widehat{H}=\widehat{H}_{2}+\widehat{\mathcal{H}}_{3}+\widehat{\mathcal{H}}_{4}+\cdots$, причем $\left[\widehat{\mathcal{A}} \widehat{H}, \widehat{H}_{2}\right]=0$.

Настоящая работа построена следующим образом. В разделе 2 вводятся основные понятия и конструкции из работы [5]. Почти все теоремы в этом разделе даны без доказательства. Доказательство вспомогательного результата дано в приложении.

В разделе 3 доказана основная теорема о приведении квантовой наблюдаемой к нормальной форме. В п. 3.1 квадратичная квантовая наблюдаемая приводится к нормальной форме линейной заменой. В п. 3.2 доказана основная теорема, сформулированная выше. В разделе 4 установлено несколько важных следствий и уточнений этой теоремы. В п. 4.1 построен явный вид нормальной формы в случае нерезонансных частот, в п. 4.2 - эрмитова нормальная форма для эрмитовой наблюдаемой. В п. 4.3 рассматриваются вопросы единственности нормальной формы и нормализующего преобразования. 


\section{2. НЕКОММУТАТИВНЫЕ НАБЛЮДАЕМЫЕ}

2.1. Алгебра $\widehat{\mathbb{H}}$. Введем некоторые важные понятия и обозначения. Пусть $\hat{z}$ есть моном (1). Число $k$ называется степенью монома. Будем говорить, что моном $\hat{z}$ имеет тип $(\mu, \nu) \in \mathbb{Z}_{+}^{2 n}$, записывая это следующим образом:

$$
\text { type } \hat{z}=(\mu, \nu), \quad \mu=\left(\mu_{1}, \ldots, \mu_{n}\right), \quad \nu=\left(\nu_{1}, \ldots, \nu_{n}\right),
$$

если он содержит в точности $\mu_{j}$ раз множитель $\hat{p}_{j}$ и $\nu_{j}$ раз множитель $\hat{x}_{j}$ для всех $j=1, \ldots, n$.

Пусть $\widehat{\mathbb{O}}$, как и ранее, алгебра (2). Рассмотрим следующие конечномерные подпространства однородных форм в $\widehat{\mathbb{O}}$ :

$$
\widetilde{\mathbb{F}}_{k}=\left\{\widehat{F}=\sum_{\operatorname{deg} \hat{z}=k} f_{\hat{z}} \hat{z}\right\} \subset \widehat{\mathbb{O}}, \quad \widetilde{\mathbb{F}}^{\mu, \nu}=\left\{\widehat{F}=\sum_{\text {type } \hat{z}=(\mu, \nu)} g_{\hat{z}} \hat{z}\right\} \subset \widehat{\mathbb{O}} .
$$

Положим, как и ранее, $\mathbf{r}_{j}:=\hat{p}_{j} \circ \hat{x}_{j}-\hat{x}_{j} \circ \hat{p}_{j}$ и рассмотрим идеал $\hat{J} \subset \widehat{\mathbb{O}}$, порожденный левыми частями тождеств (3)-(5).

Рассмотрим фактор-алгебру $\widehat{\mathbb{H}}$ :

$$
\widehat{\mathbb{H}}=\widehat{\mathbb{O}} / \hat{J}, \quad \pi: \widehat{\mathbb{O}} \mapsto \widehat{\mathbb{H}}, \quad \mathbf{r}=\pi\left(\mathbf{r}_{1}\right)=\cdots=\pi\left(\mathbf{r}_{n}\right),
$$

где $\pi$ - естественный гомоморфизм проекции. Для упрощения записи будем обозначать элементы $\pi \hat{p}_{j}$ и $\pi \hat{x}_{j}$ в алгебре $\widehat{\mathbb{H}}$ через $\hat{p}_{j}$ и $\hat{x}_{j}$ соответственно.

Обозначим линейные пространства однородных форм в алгебре $\widehat{\mathbb{H}}$ следующим образом:

$$
\mathbb{F}_{k}=\pi \widetilde{\mathbb{F}}_{k}, \quad \mathbb{F}^{\mu, \nu}=\pi \mathbb{F}^{\mu, \nu} .
$$

Из тождеств, порождающих $\widehat{\mathbb{H}}$, ясно, что $\mathbb{F}_{k} \cap \mathbb{F}_{l}=\{0\}$ для всех $k \neq l$. Для пространств $\mathbb{F}^{\mu, \nu}$ аналогичное утверждение не выполнено. Вообще говоря, неверно, что $\mathbb{F}^{\mu, \nu} \cap \mathbb{F}^{\mu^{\prime}, \nu^{\prime}}=\{0\}$ для разных векторов $(\mu, \nu) \in \mathbb{Z}_{+}^{2 n}$ и $\left(\mu^{\prime}, \nu^{\prime}\right) \in \mathbb{Z}_{+}^{2 n}$. Пусть, например, $n=2$. Положим

$$
\mu=\nu=(1,0), \quad \mu^{\prime}=\nu^{\prime}=(0,1) .
$$

Тогда $\mathbf{r} \in \mathbb{F}^{\mu, \nu} \cap \mathbb{F}^{\mu^{\prime}, \nu^{\prime}}$. Более подробно свойства пространств $\mathbb{F}^{\mu, \nu}$ исследованы ниже в п. 3.3 .

Если $\widehat{F} \in \bigoplus_{j=k}^{\infty} \mathbb{F}_{j}$, мы используем следующее обозначение: $\widehat{F}=O_{k}(\hat{p}, \hat{x})$.

Далее, пусть $\hat{J}_{0}$ есть идеал в алгебре $\widehat{\mathbb{H}}$, порожденный $\mathbf{r}$. Тогда фактор-алгебру $\widehat{\mathbb{H}} / \hat{J}_{0}$ можно отождествить с коммутативной алгеброй классических формальных рядов Тейлора с комплексными коэффициентами. Назовем ее алгеброй классических наблюдаемых и обозначим через $\mathbb{O}$. Естественный гомоморфизм проекции обозначим aver: $\widehat{\mathbb{H}} \rightarrow \mathbb{O}$ и назовем усреднением. Этот гомоморфизм отображает некоторые квантовые наблюдаемые в одну классическую. Например,

$$
\operatorname{aver}\left(\hat{p} \circ \hat{x}^{2}\right)=\operatorname{aver}(\hat{x} \circ \hat{p} \circ \hat{x})=\operatorname{aver}\left(\hat{x}^{2} \circ \hat{p}\right)=p x^{2} .
$$

Образующие в алгебре $\mathbb{D}$ обозначаются как $p_{j}=\operatorname{aver} \hat{p}_{j}, x_{j}=\operatorname{aver} \hat{x}_{j}$.

В ассоциативной алгебре $\widehat{\mathbb{H}}$ можно ввести еще одну операцию $\widehat{F}, \widehat{G} \mapsto[\widehat{F}, \widehat{G}]$, называемую коммутатором. 
Для наблюдаемых $\widehat{F}, \widehat{G} \in \widehat{\mathbb{H}}$ положим $\widehat{H}=\widehat{F} \circ \widehat{G}-\widehat{G} \circ \widehat{F}$. Тогда aver $\widehat{H}=0$, и, следовательно, $\widehat{H} \in \hat{J}_{0}$. Таким образом, $\widehat{H}=\mathbf{r} \circ \widehat{H}_{0}$, где наблюдаемая $\widehat{H}_{0}$ определена единственным образом, поскольку в алгебре $\widehat{\mathbb{H}}$ отсутствуют нетривиальные делители нуля. Определим операцию $\widehat{F}, \widehat{G} \mapsto[\widehat{F}, \widehat{G}]=\widehat{H}_{0}$, называемую коммутатором. Неформально он определяется следующим образом:

$$
[\widehat{F}, \widehat{G}]=\frac{\widehat{F} \circ \widehat{G}-\widehat{G} \circ \widehat{F}}{\mathbf{r}} .
$$

В дальнейшем часто используем стандартное обозначение

$$
\operatorname{ad}_{\widehat{F}} \widehat{G}=[\widehat{F}, \widehat{G}],
$$

где ad называется коприсоединенным представлением.

Легко увидеть, что коммутатор удовлетворяет тождествам Лейбница и Якоби (cм. [5]):

$$
[\widehat{F} \circ \widehat{G}, \widehat{H}]=\widehat{F} \circ[\widehat{G}, \widehat{H}]+[\widehat{F}, \widehat{H}] \circ \widehat{G}, \quad[[\widehat{F}, \widehat{G}], \widehat{H}]+[[\widehat{G}, \widehat{H}], \widehat{F}]+[[\widehat{H}, \widehat{F}], \widehat{G}]=0
$$

Введенная нами операция $[\cdot, \cdot]$ задает на $\widehat{\mathbb{H}}$ структуру алгебры Ли. Также можно рассматривать $\mathbb{O}$ как алгебру Ли относительно стандартной скобки Пуассона:

$$
F, G \mapsto\{F, G\}=\sum_{j=1}^{n} \frac{\partial F}{\partial p_{j}} \frac{\partial G}{\partial x_{j}}-\frac{\partial F}{\partial x_{j}} \frac{\partial G}{\partial p_{j}}
$$

Оказывается, усреднение aver является гомоморфизмом алгебр Ли $\widehat{\mathbb{H}}_{n}$ и $\mathbb{O}_{n}[5]$.

2.2. r-Разложение и базис $\mathbf{E}_{s}$.

ПредлоЖЕНИЕ 1. Всякая наблюдаемая $\widehat{F} \in \widehat{\mathbb{H}}$ представляется единственным образом в виде следующего разложения:

$$
\widehat{F}=\sum_{\substack{k \in \mathbb{Z}_{+},(\mu, \nu) \in \mathbb{Z}_{+}^{2 n}}} C_{\mu, \nu, k} \mathbf{r}^{k} \circ \hat{p}_{1}^{\mu_{1}} \circ \cdots \circ \hat{p}_{n}^{\mu_{n}} \circ \hat{x}_{1}^{\nu_{1}} \circ \cdots \circ \hat{x}_{n}^{\nu_{n}}=\sum_{k, \mu, \nu} \mathbf{r}^{k} \circ \hat{p}^{\mu} \circ \hat{x}^{\nu} .
$$

Доказательство предложения 1 можно найти в работе [5].

Пусть $n=1$. Положим $\hat{\eta}:=\hat{p} \circ \hat{x}, \hat{\zeta}:=\hat{x} \circ \hat{p}$. Рассмотрим следующее множество B $\mathbb{F}^{s, s}$ :

$$
\mathbf{E}_{s}=\left\{\hat{z}_{1} \circ \cdots \circ \hat{z}_{s} \mid \hat{z}_{j} \in\{\hat{\eta}, \hat{\zeta}\}\right\}
$$

Поскольку $\hat{\eta}$ и $\hat{\zeta}$ коммутируют, то не более чем $s+1$ элементов этого множества различны.

ПрЕДЛОЖЕНИЕ 2. Множество $\mathbf{E}_{s}$ есть базис в векторном пространстве $\mathbb{F}^{s, s}$.

ДокАзАТЕЛЬство. Из результатов работы [5] следует, что размерность пространства $\mathbb{F}^{s, s}$ равна $s+1$. Например, в качестве базиса можно взять элементы

$$
\hat{p}^{s} \circ \hat{x}^{s}, \quad \hat{p}^{s-1} \circ \hat{x}^{s} \circ \hat{p}, \quad \ldots, \quad \hat{p} \circ \hat{x}^{s} \circ \hat{p}^{s-1}, \quad \hat{x}^{s} \circ \hat{p}^{s} .
$$


Тогда достаточно доказать, что любой элемент этого векторного пространства представим в виде линейной комбинации элементов $\mathbf{E}_{s}$. Докажем это с помощью индукции по $s$. Если $s=1$, то утверждение очевидно. Пусть $\widehat{F} \in \mathbb{F}^{s, s}$. Достаточно рассмотреть случай, когда $\widehat{F}$ - моном. Не ограничивая общности, можно считать, что

$$
\widehat{F}=\hat{p}^{\alpha} \circ \hat{x}^{\beta} \circ \widehat{G}, \quad \alpha, \beta \neq 0,
$$

где $\widehat{G}$ - моном степени, меньшей $2 s$. Тогда из соотношения

$$
\hat{p}^{\alpha-1} \circ \hat{x}=\hat{x} \circ \hat{p}^{\alpha-1}+(\alpha-1) \mathbf{r} \circ \hat{p}^{\alpha-2}
$$

следует, что $\widehat{F}=\hat{p} \circ \hat{x} \circ \widehat{G}_{1}+\hat{x} \circ \hat{p} \circ \widehat{G}_{2}$, где

$$
\widehat{G}_{1}=\hat{p}^{\alpha-1} \circ \hat{x}^{\beta-1} \circ \widehat{G}+(\alpha-1) \hat{p}^{\alpha-2} \circ \hat{x}^{\beta-1} \circ \widehat{G}, \quad \widehat{G}_{2}=-(\alpha-1) \hat{p}^{\alpha-2} \circ \hat{x}^{\beta-1} \circ \widehat{G} .
$$

По предположению индукции $\widehat{G}_{1}$ и $\widehat{G}_{2}$ раскладываются по базису $\mathbf{E}_{s-1}$. Следовательно, $\widehat{F}$ раскладывается по базису $\mathbf{E}_{s}$. Предложение доказано.

2.3. Эрмитовы наблюдаемые. Для любого монома в алгебре $\widehat{\mathbb{O}}$, имеющего вид $\hat{z}=\hat{z}_{1} \circ \cdots \circ \hat{z}_{k} \in \mathbb{F}_{k}$, и постоянной $\alpha \in \mathbb{C}$ определим следующую операцию:

$$
(\alpha \hat{z})^{*}=\bar{\alpha} \hat{z}_{k} \circ \cdots \circ \hat{z}_{1}
$$

называемую эрмитовым сопряжением или просто сопряжением. Эту операцию можно продолжить по линейности до инволюции в $\widehat{\mathbb{O}}$. Пусть теперь $\widehat{F} \in \widehat{\mathbb{H}}$. Возьмем произвольный элемент $\widehat{G} \in \widehat{\mathbb{O}}$ такой, что $\pi \widehat{G}=\widehat{F}$. Положим $\widehat{F}^{*}=\pi\left(\widehat{G}^{*}\right)$. Как показано в работе [5], такое определение корректно и задает инволюцию в $\widehat{\mathbb{H}}$.

Операция эрмитова сопряжения удовлетворяет следующим свойствам:

$(\alpha \widehat{F}+\beta \widehat{G})^{*}=\bar{\alpha} \widehat{F}^{*}+\bar{\beta} \widehat{G}^{*}, \quad(\widehat{F} \circ \widehat{G})^{*}=\widehat{G}^{*} \circ \widehat{F}^{*}, \quad[\widehat{F}, \widehat{G}]^{*}=\left[\widehat{F}^{*}, \widehat{G}^{*}\right], \quad \mathbf{r}^{*}=-\mathbf{r}$ для всех $\widehat{F}, \widehat{G} \in \widehat{\mathbb{H}}$.

Наблюдаемая $\widehat{F}$ называется самосопряженной или эрмитовой, если $\widehat{F}^{*}=\widehat{F}$.

2.4. Автоморфизмы алгебры $\widehat{\mathbb{H}}$. Рассмотрим наблюдаемые

$$
\begin{aligned}
& \widehat{P}_{j}=c_{j, 1} \hat{p}_{1}+\cdots+c_{j, n} \hat{p}_{n}+c_{j, n+1} \hat{x}_{1}+\cdots+c_{j, 2 n} \hat{x}_{n}+O_{2}(\hat{p}, \hat{x}), \\
& \widehat{X}_{j}=c_{j+n, 1} \hat{p}_{1}+\cdots+c_{j+n, n} \hat{p}_{n}+c_{j+n, n+1} \hat{x}_{1}+\cdots+c_{j+n, 2 n} \hat{x}_{n}+O_{2}(\hat{p}, \hat{x}),
\end{aligned}
$$

где $j=1, \ldots, n$ такие, что для всех $1 \leqslant j, k \leqslant n$

$$
\left[\widehat{P}_{j}, \widehat{P}_{k}\right]=\left[\widehat{X}_{j}, \widehat{X}_{k}\right]=0, \quad\left[\widehat{P}_{j}, \widehat{X}_{k}\right]=\delta_{j k}= \begin{cases}0, & j \neq k \\ 1, & j=k\end{cases}
$$

В частности, матрица $C=\left(c_{i, j}\right)_{i, j=1}^{2 n}$ является симплектической, т.е. $C^{\mathrm{T}} I C=I$, где

$$
I=\left(\begin{array}{cc}
0 & -E_{n \times n} \\
E_{n \times n} & 0
\end{array}\right)
$$

есть симплектическая единица. 
Определим следующее отображение:

$$
\widehat{\mathcal{A}}: \widehat{\mathbb{H}} \rightarrow \widehat{\mathbb{H}}, \quad \widehat{F}(\hat{p}, \hat{x}) \mapsto \widehat{\mathcal{A}}(\widehat{F})(\hat{p}, \hat{x})=\widehat{F}(\widehat{P}(\hat{p}, \hat{x}), \widehat{X}(\hat{p}, \hat{x})) .
$$

Оказывается, что $\widehat{\mathcal{A}}$ является автоморфизмом ассоциативной алгебры и алгебры Ли $(\widehat{\mathbb{H}}, \circ,[\cdot, \cdot]),[5]$. Это означает, что $\widehat{\mathcal{A}}$ есть биективное отображение $\widehat{\mathbb{H}}$ в себя со следующими свойствами:

$$
\widehat{\mathcal{A}}(\alpha \widehat{F}+\beta \widehat{G})=\alpha \widehat{\mathcal{A}} \widehat{F}+\beta \widehat{\mathcal{A}} \widehat{G}, \quad \widehat{\mathcal{A}}(\widehat{F} \circ \widehat{G})=\widehat{\mathcal{A}} \widehat{F} \circ \widehat{\mathcal{A}} \widehat{G}, \quad \widehat{\mathcal{A}}[\widehat{F}, \widehat{G}]=[\widehat{\mathcal{A}} \widehat{F}, \widehat{\mathcal{A}} \widehat{G}]
$$

для всех $\widehat{F}, \widehat{G} \in \widehat{\mathbb{H}}$. Как следствие получаем, что $\widehat{\mathcal{A}} \mathbf{r}=\mathbf{r}$. В дальнейшем автоморфизмы ассоциативной алгебры и алгебры Ли $\widehat{\mathbb{H}}$ будем называть просто автоморфизмами. Эти автоморфизмы образуют группу, обозначаемую Aut $\widehat{\mathbb{H}}$, с операцией композиции, заданной следующим образом:

$$
(\widehat{\mathcal{A}} \widehat{\mathcal{B}}) \widehat{F}:=\widehat{\mathcal{A}}(\widehat{\mathcal{B}} \widehat{F}), \quad \widehat{\mathcal{A}}, \widehat{\mathcal{B}} \in \operatorname{Aut} \widehat{\mathbb{H}}, \quad \widehat{F} \in \widehat{\mathbb{H}} .
$$

Приведем два важных класса автоморфизмов. Сначала рассмотрим автоморфизм, который порождается наблюдаемыми

$$
\begin{aligned}
\widehat{P}_{j} & =c_{j, 1} \hat{p}_{1}+\cdots+c_{j, n} \hat{p}_{n}+c_{j, n+1} \hat{x}_{1}+\cdots+c_{j, 2 n} \hat{x}_{n}, \\
\widehat{X}_{j} & =c_{j+n, 1} \hat{p}_{1}+\cdots+c_{j+n, n} \hat{p}_{n}+c_{j+n, n+1} \hat{x}_{1}+\cdots+c_{j+n, 2 n} \hat{x}_{n},
\end{aligned}
$$

где $C=\left(c_{i, j}\right)_{i, j=1}^{2 n}-$ симплектическая матрица. Такие автоморфизмы образуют группу, которую обозначим через $\mathrm{Aut}_{L} \widehat{\mathbb{H}}$. Если наблюдаемые, задающие некоторый автоморфизм $\widehat{\mathcal{A}}$, имеют вид

$$
\widehat{P}_{j}=\hat{p}_{j}+O_{2}(\hat{p}, \hat{x}), \quad \widehat{X}_{j}=\hat{x}_{j}+O_{2}(\hat{p}, \hat{x}),
$$

то назовем $\widehat{\mathcal{A}}$ автоморфизмом, близким к тождественному. При действии на наблюдаемую он оставляет без изменения ее однородные формы степени не выше второй.

Теперь рассмотрим наблюдаемую $\widehat{H}=O_{3}(\hat{p}, \hat{x})$ и зададим следующее отображение:

$$
\widehat{F} \mapsto e^{\tau \operatorname{ad}_{\widehat{H}}}(\widehat{F})=\sum_{j=0}^{\infty} \frac{\tau^{j}}{j !} \operatorname{ad}_{\widehat{H}}^{j} \widehat{F}=\widehat{F}+\frac{1}{1 !}[\widehat{H}, \widehat{F}] \tau+\frac{1}{2 !}[\widehat{H},[\widehat{H}, \widehat{F}]] \tau^{2}+\cdots
$$

Такое отображение, называемое экспоненциальным, корректно задано и является автоморфизмом $\widehat{\mathbb{H}}$. Это аналог классического сдвига вдоль гамильтонова векторного поля с гамильтонианом $H$ на время $\tau$.

Все введенные конструкции могут быть определены и в классическом случае. Соответствующие группы автоморфизмов мы будем обозначать Aut $\mathbb{O}$, Aut $_{L} \mathbb{O}$.

\section{5. Лемма Пуанкаре для $\widehat{\mathbb{H}}$.}

ПрЕДЛОЖЕНИЕ 3. Пусть $\widehat{P}_{1}, \ldots, \widehat{P}_{n}, \widehat{X}_{1}, \ldots, \widehat{X}_{n} \in \widehat{\mathbb{H}}_{n}$ удовлетворяют соотношениям

$$
\operatorname{ad}_{\hat{p}_{j}} \widehat{P}_{k}-\operatorname{ad}_{\hat{p}_{k}} \widehat{P}_{j}=0, \quad \operatorname{ad}_{\hat{x}_{j}} \widehat{X}_{k}-\operatorname{ad}_{\hat{x}_{k}} \widehat{X}_{j}=0, \quad \operatorname{ad}_{\hat{p}_{j}} \widehat{X}_{k}-\operatorname{ad}_{\hat{x}_{k}} \widehat{P}_{j}=0 .
$$

Тогда существует наблюдаемая $\widehat{W} \in \widehat{\mathbb{H}}_{n}$ такая, что

$$
\widehat{P}_{j}=\operatorname{ad}_{\widehat{W}} \hat{p}_{j}, \quad \widehat{X}_{j}=\operatorname{ad}_{\widehat{W}} \hat{x}_{j} .
$$


ЗАмечАниЕ 1. Применяя усреднение aver, получаем из этого утверждения следующий результат. Форма $\omega=P_{1} d x_{1}+\cdots+P_{n} d x_{n}-X_{1} d p_{1}-\cdots-X_{n} d p_{n}$ в окрестности нуля замкнута тогда и только тогда, когда она точна. Это частный случай классического утверждения, которое носит название леммы Пуанкаре.

Доказательство предложения 3 приведено в приложении.

ПРЕДЛОЖЕНИЕ 4 (О ПРИБЛИЖЕНИИ АВТОМОРФИЗМА). Для любого автоморфизма существуют $\widehat{W}_{k} \in \mathbb{F}_{k}$ такие, что

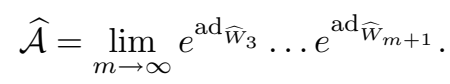

ЗАмечАние 2. Предложение 4 носит формальный характер. Понимать его следует таким образом: для любой наблюдаемой $\widehat{F} \in \widehat{\mathbb{H}}$

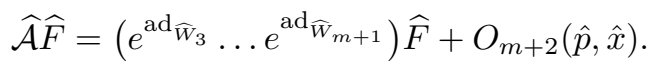

ДокАЗАТЕЛЬСтво. Пусть автоморфизм $\widehat{\mathcal{A}} \in \mathrm{Aut} \widehat{\mathbb{H}}$ порождается наблюдаемыми

$$
\begin{array}{ll}
\widehat{P}_{j}=\hat{p}_{j}+\widehat{F}_{2}^{(j)}+\widehat{F}_{3}^{(j)}+\cdots+\widehat{F}_{m}^{(j)}+O_{m+1}(\hat{p}, \hat{x}), & \widehat{F}_{k}^{(j)} \in \mathbb{F}_{k}, \\
\widehat{X}_{j}=\hat{x}_{j}+\widehat{G}_{2}^{(j)}+\widehat{G}_{3}^{(j)}+\cdots+\widehat{G}_{m}^{(j)}+O_{m+1}(\hat{p}, \hat{x}), & \widehat{G}_{k}^{(j)} \in \mathbb{F}_{k} .
\end{array}
$$

По определению автоморфизма выполнены следующие равенства:

$$
\left[\hat{p}_{j}, \widehat{G}_{2}^{k}\right]+\left[\widehat{F}_{2}^{j}, \hat{x}_{k}\right]=0, \quad\left[\hat{p}_{j}, \widehat{F}_{2}^{k}\right]+\left[\widehat{F}_{2}^{j}, \hat{p}_{k}\right]=0, \quad\left[\hat{x}_{j}, \widehat{G}_{2}^{k}\right]+\left[\widehat{G}_{2}^{j}, \hat{x}_{k}\right]=0 .
$$

В соответствии с предложением 3 для $\widehat{\mathbb{H}}$ существует наблюдаемая $\widehat{W}_{3} \in \mathbb{F}_{3}$ такая, что

$$
\widehat{F}_{2}^{(j)}=\operatorname{ad}_{\widehat{W}_{3}} \hat{p}_{j}, \quad \widehat{G}_{2}^{(j)}=\operatorname{ad}_{\widehat{W}_{3}} \hat{x}_{j} .
$$

Следовательно, автоморфизм $e^{\operatorname{ad}_{\widehat{W}_{3}}}$ порождается наблюдаемыми

$$
\widehat{P}_{j}=\hat{p}_{j}+\widehat{F}_{2}^{(j)}+\hat{f}_{3}^{(j)}+O_{4}(\hat{p}, \hat{x}), \quad \widehat{X}_{j}=\hat{x}_{j}+\widehat{G}_{2}^{(j)}+\hat{g}_{3}^{(j)}+O_{4}(\hat{p}, \hat{x}) .
$$

По определению автоморфизма имеем соотношения

$$
\left[\hat{p}_{j}, \widehat{G}_{3}^{k}\right]+\left[\widehat{F}_{2}^{j}, \widehat{G}_{2}^{k}\right]+\left[\widehat{F}_{3}^{j}, \hat{x}_{k}\right]=0, \quad\left[\hat{p}_{j}, \hat{g}_{3}^{k}\right]+\left[\widehat{F}_{2}^{j}, \widehat{G}_{2}^{k}\right]+\left[\hat{f}_{3}^{j}, \hat{x}_{k}\right]=0
$$

Следовательно, $\left[\hat{p}_{j}, \hat{g}_{3}^{k}-\widehat{G}_{3}^{k}\right]+\left[\hat{f}_{3}^{j}-\widehat{F}_{3}^{j}, \hat{x}_{k}\right]=0$. Аналогично получаются соотношения

$$
\left[\hat{p}_{j}, \hat{f}_{3}^{k}-\widehat{F}_{3}^{k}\right]+\left[\hat{f}_{3}^{j}-\widehat{F}_{3}^{j}, \hat{p}_{k}\right]=0, \quad\left[\hat{x}_{j}, \hat{g}_{3}^{k}-\widehat{G}_{3}^{k}\right]+\left[\hat{g}_{3}^{j}-\widehat{G}_{3}^{j}, \hat{x}_{k}\right]=0 .
$$

В соответствии с предложением 3 для $\widehat{\mathbb{H}}$ существует наблюдаемая $\widehat{W}_{4} \in \mathbb{F}_{3}$ такая, что

$$
\widehat{F}_{3}^{(j)}=\hat{f}_{3}^{j}+\operatorname{ad}_{\widehat{W}_{4}} \hat{p}_{j}, \quad \widehat{G}_{3}^{(j)}=\hat{g}_{3}^{j}+\operatorname{ad}_{\widehat{W}_{4}} \hat{x}_{j} .
$$

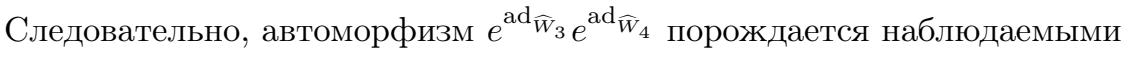

$$
\hat{P}_{j}=\hat{p}_{j}+\widehat{F}_{2}^{(j)}+\widehat{F}_{3}^{(j)}+\hat{f}_{4}^{(j)}+O_{5}(\hat{p}, \hat{x}), \quad \hat{X}_{j}=\hat{x}_{j}+\widehat{G}_{2}^{(j)}+\widehat{G}_{3}^{(j)}+\hat{g}_{4}^{(j)}+O_{5}(\hat{p}, \hat{x}) .
$$

Продолжая эту процедуру, получаем требуемое утверждение. Предложение доказано. 


\section{3. НОРМАЛЬНАЯ ФОРМА КВАНТОВЫХ НАБЛЮДАЕМЫХ}

3.1. Нормальная форма квадратичных наблюдаемых. Рассмотрим квадратичную квантовую наблюдаемую $\widehat{H}_{2} \in \mathbb{F}_{2}$. Построим такой автоморфизм $\widehat{\mathcal{A}} \in$ $\mathrm{Aut}_{L} \widehat{\mathbb{H}}$, который приводил бы $\widehat{H}_{2}$ к наиболее простому виду.

Для начала мы рассмотрим классическую квадратичную форму или гамильтониан $H_{2}=\langle\Omega z, z\rangle / 2$, где $z=\left(p_{1}, \ldots, p_{n}, x_{1}, \ldots, x_{n}\right)^{\mathrm{T}}$ - вектор-столбец, $\Omega=\Omega^{\mathrm{T}}-$ симметричная $(2 n \times 2 n)$-матрица, а $\langle\cdot, \cdot\rangle$, как и ранее, есть стандартное скалярное произведение. По определению собственные значения гамильтониана $\widehat{H}_{2}$ суть собственные значения матрицы $I \Omega$, где $I$ - симплектическая единица (7). Будем говорить, что собственные значения $\widehat{H}_{2}$ просты, если матрица $I \Omega$ диагонализируема.

Tеорема 1. Пусть $\widehat{H}_{2} \in \mathbb{F}_{2}$ и собственные значения $\lambda_{1}, \ldots, \lambda_{n}$ гамильтониана aver $\widehat{H}_{2}$ просты. Тогда существует автоморфизм $\widehat{\mathcal{A}} \in \mathrm{Aut}_{L} \widehat{\mathbb{H}}$ такой, что

$$
\widehat{\mathcal{A}} \widehat{H}_{2}=\sum_{j=1}^{n} \lambda_{j} \hat{p}_{j} \circ \hat{x}_{j}+c \mathbf{r}, \quad c \in \mathbb{C} .
$$

ДокАЗАтеЛЬСтво. Рассмотрим классическую квадратичную форму $H_{2}=$ aver $\widehat{H}_{2}$. Хорошо известно, что существует классическая линейная замена $\mathcal{A} \in$ $\mathrm{Aut}_{L} \mathbb{O}$, порожденная наблюдаемыми,

$$
Z=C z, \quad z=\left(p_{1}, \ldots, p_{n}, x_{1}, \ldots, x_{n}\right), \quad Z=\left(P_{1}, \ldots, P_{n}, X_{1}, \ldots, X_{n}\right),
$$

для некоторой симплектической матрицы $C$ такая, что

$$
\mathcal{A} H_{2}=\sum_{j=1}^{n} \lambda_{j} p_{j} x_{j}
$$

Тогда автоморфизм $\widehat{\mathcal{A}} \in \mathrm{Aut}_{L} \widehat{\mathbb{H}}$, порожденный той же самой матрицей,

$$
\widehat{Z}=C \hat{z}, \quad \hat{z}=\left(\hat{p}_{1}, \ldots, \hat{p}_{n}, \hat{x}_{1}, \ldots, \hat{x}_{n}\right), \quad \widehat{Z}=\left(\widehat{P}_{1}, \ldots, \widehat{P}_{n}, \widehat{X}_{1}, \ldots, \widehat{X}_{n}\right),
$$

приводит $\widehat{H}$ к виду (11). Теорема доказана.

\section{2. Основная теорема.}

Teорема 2. Пусть $\widehat{H}=\widehat{H}_{2}+O_{3}(\hat{p}, \hat{x}) \in \widehat{\mathbb{H}}$, где $\widehat{H}_{2}$ имеет вид правой части (11). Тогда существует автоморфизм $\widehat{\mathcal{A}} \in \mathrm{Aut} \widehat{\mathbb{H}}$, близкий $\kappa$ тождественному и такой, что

$$
\left[\widehat{H}_{2}, \widehat{\mathcal{A}} \widehat{H}\right]=0 .
$$

Наблюдаемая $\widehat{\mathcal{H}}=\widehat{\mathcal{A}} \widehat{H}$, удовлетворяющая (14), называется нормальной формой. Вектор $\lambda=\left(\lambda_{1}, \ldots, \lambda_{n}\right)$ называется вектором частот. Введем обозначения

$$
\begin{aligned}
R(\lambda) & =\left\{(\mu, \nu) \mid \mu, \nu \in \mathbb{Z}_{+}^{n},\langle\mu-\nu, \lambda\rangle=0\right\} \\
\widehat{\mathbb{N}}_{\lambda} & =\bigcup_{(\mu, \nu) \in R(\lambda)} \mathbb{F}^{\mu, \nu}, \quad \widehat{\mathbb{N}}_{\lambda}^{c}=\bigcup_{(\mu, \nu) \notin R(\lambda)} \mathbb{F}^{\mu, \nu} .
\end{aligned}
$$


Пространство $\widehat{\mathbb{N}}_{\lambda}$ является ассоциативной подалгеброй в $\widehat{\mathbb{H}}$, а пространство $\widehat{\mathbb{N}}_{\lambda}^{c}-$ линейным подпространством там же. Ниже (см. следствие 1) мы покажем, что наблюдаемые из $\widehat{\mathbb{N}}_{\lambda}$ и только они удовлетворяют соотношению (14). Поэтому мы назовем $\widehat{\mathbb{N}}_{\lambda}$ алгеброй нормальных форм.

Частоты $\lambda$ называются резонансными, если существуют такие числа $k_{1}, \ldots, k_{n} \in$ $\mathbb{Z}$, не равные нулю одновременно, что $\sum_{j=1}^{n} k_{j} \lambda_{j}=0$. В противном случае вектор частот называется нерезонансным.

Заметим, что множество $R(\lambda)$, введенное выше, содержит векторы вида $(\mu, \mu)$ для всех векторов частот $\lambda$. Для нерезонансного вектора частот $\lambda$ множество $R(\lambda)$ состоит лишь из векторов такого вида. В нерезонансном случае ассоциативная алгебра $\widehat{\mathbb{N}}_{\lambda}$ коммутативна (одинаковая для всех векторов $\lambda$ ), в резонансном - некоммутативна.

3.3. Свойства пространств однородных форм. Прежде чем приступать к доказательству основной теоремы, получим некоторые свойства пространств $\mathbb{F}^{\mu, \nu}$, $\mathbb{N}_{\lambda}$ и $\mathbb{N}_{\lambda}^{c}$.

Для всякого целочисленного вектора $d \in \mathbb{Z}_{+}^{n}$ положим $\|d\|=\sum_{j=1}^{n} d_{j}$. Введем обозначение

$$
\mathbb{F}_{-}^{\mu, \nu}=\bigcup_{d \in \mathbb{Z}_{+}^{n},\|d\|=1} \mathbb{F}^{\mu-d, \nu-d},
$$

где формально положим $\mathbb{F}^{-1, \nu}=\mathbb{F}^{\mu,-1}=\{0\}$.

ПреДЛОЖениЕ 5. Пустъ $\widehat{F} \in \mathbb{F}^{\mu, \nu}$ u $\widehat{F}=\mathbf{r} \circ \widehat{G}$. Тогда $\widehat{G} \in \mathbb{F}_{-}^{\mu, \nu}$.

ДокаЗАтельство. Пусть $\mu^{\prime}, \nu^{\prime} \in \mathbb{Z}_{+}^{n}, \gamma \in \mathbb{Z}_{+}$. Запись $\left(\mu^{\prime}, \nu^{\prime}, \gamma\right) \in M_{\mu, \nu}$ означает, что существуют такие векторы $\mu^{0}, \nu^{0} \in \mathbb{Z}_{+}^{n}$, что

$$
\mu=\mu^{\prime}+\mu^{0}, \quad \nu=\nu^{\prime}+\nu^{0}, \quad\left\|\mu^{0}\right\|=\left\|\nu^{0}\right\|=\gamma .
$$

Согласно предложению 1 наблюдаемую $\widehat{F}$ можно единственным образом представить в виде

$$
\widehat{F}=\mathbf{r} \circ \sum_{\left(\mu^{\prime}, \nu^{\prime}, \gamma\right) \in M_{\mu, \nu}} C_{\mu^{\prime}, \nu^{\prime}, \gamma} \mathbf{r}^{\gamma-1} \circ \hat{p}^{\mu^{\prime}} \circ \hat{x}^{\nu^{\prime}},
$$

где каждое слагаемое лежит в некотором подпространстве $\mathbb{F}^{\mu-d, \nu-d}, d \in \mathbb{Z}_{+}^{n}$ и $\|d\|=1$. Предложение доказано.

Пусть $\mu^{\prime}, \nu^{\prime}, \mu^{\prime \prime}, \nu^{\prime \prime} \in \mathbb{Z}_{+}^{n}$. Тогда $\left(\mu^{\prime}, \nu^{\prime}\right) \sim\left(\mu^{\prime \prime}, \nu^{\prime \prime}\right)$, если существуют такие векторы $l^{\prime}, l^{\prime \prime}, \mu, \nu \in \mathbb{Z}_{+}^{n}$, что

$$
\mu^{\prime \prime}=\mu+l^{\prime \prime}, \quad \nu^{\prime \prime}=\nu+l^{\prime \prime}, \quad \mu^{\prime}=\mu+l^{\prime}, \quad \nu^{\prime}=\nu+l^{\prime}, \quad\left\|l^{\prime}\right\|=\left\|l^{\prime \prime}\right\| .
$$

ПреДЛОЖениЕ 6. Соотношение $\mathbb{F}^{\mu^{\prime}, \nu^{\prime}} \cap \mathbb{F}^{\mu^{\prime \prime}, \nu^{\prime \prime}} \neq\{0\}$ справедливо тогда и толъко тогда, когда $\left(\mu^{\prime}, \nu^{\prime}\right) \sim\left(\mu^{\prime \prime}, \nu^{\prime \prime}\right)$.

ДокАЗАТЕЛЬство. Достаточность. Пусть выполнено (15), причем $\left\|l^{\prime}\right\|=\left\|l^{\prime \prime}\right\|=$ $\gamma$. Тогда

$$
\mathbf{r}^{\gamma} \circ \hat{p}^{\mu} \circ \hat{x}^{\nu} \in \mathbb{F}^{\mu^{\prime}, \nu^{\prime}} \cap \mathbb{F}^{\mu^{\prime \prime}, \nu^{\prime \prime}}
$$

4 Теоретическая и математическая физика, т. 160, № 3, 2009 г. 
Необходимость. Пусть $\mathbb{F}^{\mu^{\prime}, \nu^{\prime}} \cap \mathbb{F}^{\mu^{\prime \prime}, \nu^{\prime \prime}} \neq\{0\}$. Тогда существует такое число $k \in \mathbb{Z}_{+}$, что $\mathbb{F}^{\mu^{\prime}, \nu^{\prime}} \cup \mathbb{F}^{\mu^{\prime \prime}, \nu^{\prime \prime}} \subset \mathbb{F}_{k}$. Докажем утверждение от противного индукцией по $k$.

Для $k \leqslant 2$ утверждение, очевидно, выполнено. Предположим, что пары $\left(\mu^{\prime}, \nu^{\prime}\right)$ и $\left(\mu^{\prime \prime}, \nu^{\prime \prime}\right)$ не эквивалентны, причем $\left\|\mu^{\prime}\right\|+\left\|\nu^{\prime}\right\|=\left\|\mu^{\prime \prime}\right\|+\left\|\nu^{\prime \prime}\right\|=k$. Докажем, что $\mathbb{F}^{\mu^{\prime}, \nu^{\prime}} \cap \mathbb{F}^{\mu^{\prime \prime}, \nu^{\prime \prime}}=\{0\}$. Рассмотрим наблюдаемую $\widehat{F} \in \mathbb{F}^{\mu, \nu} \cap \mathbb{F}^{\mu^{\prime}, \nu^{\prime}}$. Тогда

$$
\text { aver } \widehat{F}=f_{0}^{\prime} p_{1}^{\mu_{1}^{\prime}} \ldots p_{n}^{\mu_{n}^{\prime}} x_{1}^{\nu_{1}^{\prime}} \ldots x_{n}^{\nu_{n}^{\prime}}=f_{0}^{\prime \prime} p_{1}^{\mu_{1}^{\prime \prime}} \ldots p_{n}^{\mu_{n}^{\prime \prime}} x_{1}^{\nu_{1}^{\prime \prime}} \ldots x_{n}^{\nu_{n}^{\prime \prime}} \text {. }
$$

Следовательно, aver $\widehat{F}=0$, или $\widehat{F}=\mathbf{r} \circ \widehat{G}$. Согласно предложению 5 имеем $\widehat{G} \in \mathbb{F}_{-}^{\mu^{\prime}, \nu^{\prime}} \cap \mathbb{F}_{-}^{\mu^{\prime \prime}, \nu^{\prime \prime}}$. По предположению индукции $\widehat{G}=0$. Предложение доказано.

Далее положим

$$
\widehat{H}_{2}=\sum_{j=1}^{n} \lambda_{j} \hat{p}_{j} \circ \hat{x}_{j} .
$$

ПредЛОЖЕНИЕ 7. Пусть $\widehat{F} \in \mathbb{F}^{\mu, \nu}$. Тогда $\left[\widehat{F}, \widehat{H}_{2}\right]=\langle\lambda, \mu-\nu\rangle \widehat{F}$.

ДокАЗАтЕльство. Достаточно рассмотреть случай, когда $\widehat{F}$ - моном. Общий случай следует из линейности. Проведем доказательство с помощью индукции по степени $k$ монома $\widehat{F}$.

Пусть $k=1$. Рассмотрим лишь случай $\widehat{F}=\hat{p}_{l}$, поскольку случай $\widehat{F}=\hat{x}_{l}$ аналогичен. Имеем

$$
\left[\hat{p}_{l}, \sum_{j=1}^{n} \lambda_{j} \hat{p}_{j} \circ \hat{x}_{j}\right]=\lambda_{l} \hat{p}_{l}=\langle\lambda, \mu-\nu\rangle \hat{p}_{l} .
$$

База индукции доказана.

Пусть $\widehat{F}_{0}-$ моном степени $k-1$. Предположим, что $\widehat{F}=\hat{p}_{l} \circ \widehat{F}_{0}$. Другие случаи $\left(\widehat{F}=\hat{x}_{l} \circ \widehat{F}_{0}, \widehat{F}=\widehat{F}_{0} \circ \hat{p}_{l}, \widehat{F}=\widehat{F}_{0} \circ \hat{x}_{l}\right)$ рассматриваются аналогично. Имеем

$$
\left[\hat{p}_{l} \circ \widehat{F}_{0}, \widehat{H}_{2}\right]=\hat{p}_{l} \circ\left[\widehat{F}_{0}, \widehat{H}_{2}\right]+\left[\hat{p}_{l}, \widehat{H}_{2}\right] \circ \widehat{F}_{0}=\left\langle\lambda, \mu^{\prime}-\nu\right\rangle \hat{p}_{l} \circ \widehat{F},
$$

где $\mu_{j}^{\prime}=\mu_{j}$ при $j \neq l$ и $\mu_{l}^{\prime}=\mu_{l}+1$. Шаг индукции доказан.

ПредлОЖЕНИЕ 8. 1. Пусть $\widehat{F} \in \widehat{\mathbb{N}}_{\lambda}$. Тогда $\operatorname{ad}_{\widehat{H}_{2}} \widehat{F}=0$.

2. Пусть $\widehat{F} \in \widehat{\mathbb{N}}_{\lambda}^{c} u \operatorname{ad}_{\widehat{H}_{2}} \widehat{F}=0$. Тогда $\widehat{F}=0$.

3. $\widehat{\mathbb{H}}=\widehat{\mathbb{N}}_{\lambda} \oplus \widehat{\mathbb{N}}_{\lambda}^{c}$.

ДокАЗАтЕльство. Утверждение 1 следует из предложения 7.

2. Для всех пар $(\mu, \nu) \in \mathbb{Z}_{+}^{2 n}$ составим множества вида $\bigcup_{\left(\mu^{\prime}, \nu^{\prime}\right) \sim(\mu, \nu)} \mathbb{F}^{\mu^{\prime}, \nu^{\prime}}$. Согласно предложению 6 эти множества либо совпадают, либо их пересечение есть $\{0\}$. Пронумеруем эти множества, считая совпадающие по одному разу, и обозначим их через $\widehat{\mathbb{K}}_{m}$, где $m=1,2, \ldots$. Таким образом, $\widehat{\mathbb{K}}_{m_{1}} \cap \widehat{\mathbb{K}}_{m_{2}}=\{0\}$, если $m_{1} \neq m_{2}$.

Представим $\widehat{F}$ в виде

$$
\widehat{F}=\sum_{m} \widehat{F}_{m}, \quad \widehat{F}_{m} \in \widehat{\mathbb{K}}_{m}
$$

Из предложения 7 следует, что $\operatorname{ad}_{\widehat{H}_{2}}: \widehat{\mathbb{K}}_{m} \rightarrow \widehat{\mathbb{K}}_{m}$. Поэтому если $\operatorname{ad}_{\widehat{H}_{2}} \widehat{F}=0$, то $\operatorname{ad}_{\widehat{H}_{2}} \widehat{F}_{m}=0$. Покажем, что $\widehat{F}_{m}=0$. Действительно, $\widehat{F}_{m}$ можно представить в следующем виде (не единственным образом):

$$
\widehat{F}_{m}=\sum_{s} \widehat{F}_{m, s}, \quad \widehat{F}_{m, s} \in \mathbb{F}^{\mu^{s}, \nu^{s}},
$$


где $\left(\mu^{s}, \nu^{s}\right) \sim(\mu, \nu)$ для всех $s$. Тогда согласно предложению 7

$$
\operatorname{ad}_{\widehat{H}_{2}} \widehat{F}_{m}=\sum_{s}\left\langle\lambda, \nu^{s}-\mu^{s}\right\rangle \widehat{F}_{m, s}=\langle\lambda, \nu-\mu\rangle \widehat{F}_{m}
$$

Отсюда следует, что $\widehat{F}_{m}=0$.

3. Очевидно, что $\widehat{\mathbb{H}}=\widehat{\mathbb{N}}_{\lambda} \cup \widehat{\mathbb{N}}_{\lambda}^{c}$. Из предложения 5 следует, что $\widehat{\mathbb{N}}_{\lambda} \cap \widehat{\mathbb{N}}_{\lambda}^{c}=\{0\}$.

Следствие $1 . \operatorname{ad}_{\widehat{H}_{2}} \widehat{F}=0 \Longleftrightarrow \widehat{F} \in \widehat{\mathbb{N}}_{\lambda}$.

ДокАЗАТЕЛЬСТво. Согласно предложению 8 для всякой наблюдаемой $\widehat{F} \in \widehat{\mathbb{H}}$ справедливо представление

$$
\widehat{F}=\widehat{F}_{0}+\widehat{F}_{1}, \quad \widehat{F}_{0} \in \widehat{\mathbb{N}}_{\lambda}, \quad \widehat{F}_{1} \in \widehat{\mathbb{N}}_{\lambda}^{c} .
$$

Следовательно, если $\operatorname{ad}_{\widehat{H}_{2}} \widehat{F}=0$, то $\operatorname{ad}_{\widehat{H}_{2}} \widehat{F}_{1}=0$. Отсюда согласно предложению 8 следует, что $\widehat{F}_{1}=0$.

СледСтвиЕ 2. Отображение $\operatorname{ad}_{\widehat{H}_{2}}: \widehat{\mathbb{N}}_{\lambda}^{c} \rightarrow \widehat{\mathbb{N}}_{\lambda}^{c}-$ изоморфизм линейного пространства в себя.

Доказательство. Если $\operatorname{ad}_{\widehat{H}_{2}} \widehat{F}=0$, то согласно предложению 8 имеем $\widehat{F}=0$. Поэтому $\operatorname{ad}_{\widehat{H}_{2}}-$ инъекция, а следовательно, $\operatorname{ad}_{\widehat{H}_{2}}-$ изоморфизм.

3.4. Доказательство теоремы 2. Определим $\widehat{\mathcal{A}}$ как предел последовательности экспоненциальных автоморфизмов:

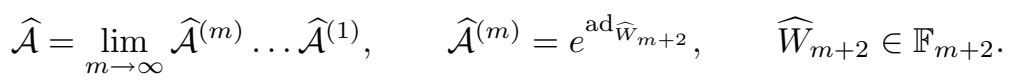

Обычно понятие предела требует введения топологии. Однако в нашем случае однородная форма любой степени стабилизируется на некотором шаге. Точнее, отображение $\widehat{\mathcal{A}}^{(m)}$ не изменяет однородные формы степени меньше, чем $m+2$.

Отображения $\widehat{\mathcal{A}}^{(m)}$ выберем так, чтобы

$$
\left(\widehat{\mathcal{A}}^{(m)} \ldots \widehat{\mathcal{A}}^{(1)}\right) \widehat{H}=\widehat{H}_{2}+\widehat{\mathcal{H}}_{3}+\cdots+\widehat{\mathcal{H}}_{m+2}+O_{m+3}(\hat{p}, \hat{x}), \quad \widehat{\mathcal{H}}_{k} \in \widehat{\mathbb{N}}_{\lambda} .
$$

Построим эти отображения индуктивно. Пусть отображения $\widehat{\mathcal{A}}^{(1)}, \ldots, \widehat{\mathcal{A}}^{(m-1)}$ построены. Преобразованную наблюдаемую обозначаем по-прежнему через $\widehat{H}$. Имеем

$$
\widehat{H}=\widehat{H}_{2}+\widehat{H}_{3}+\cdots+\widehat{H}_{m+1}+\widehat{H}_{m+2}+O_{m+3}(\hat{p}, \hat{x}), \quad \widehat{H}_{3}, \ldots, \widehat{H}_{m+1} \in \widehat{\mathbb{N}}_{\lambda} .
$$

Тогда

$\widehat{\mathcal{A}}^{(m)} \widehat{H}=\widehat{H}_{2}+\widehat{H}_{3}+\cdots+\widehat{H}_{m+1}+\widehat{\mathcal{H}}_{m+2}+O_{m+3}(\hat{p}, \hat{x}), \quad \widehat{\mathcal{H}}_{m+2}=\widehat{H}_{m+2}+\left[\widehat{W}_{m+2}, \widehat{H}_{2}\right]$.

Согласно предложению 8 имеем $\widehat{H}_{m+2}=\widehat{H}_{m+2}^{(1)}+\widehat{H}_{m+2}^{(2)}$, где $\widehat{H}_{m+2}^{(1)} \in \widehat{\mathbb{N}}_{\lambda}$ и $\widehat{H}_{m+2}^{(2)} \in \widehat{\mathbb{N}}_{\lambda}^{c}$. Согласно следствию 2 существует такая наблюдаемая $\widehat{W}_{m+2} \in \mathbb{F}_{m+2}$, что $\widehat{\mathcal{H}}_{m+2}=\widehat{H}_{m+2}^{(1)} \in \widehat{\mathbb{N}}_{\lambda}$.

Итак, построенная последовательность автоморфизмов приводит $\widehat{H}$ к нормальной форме. Теорема доказана. 


\section{4. СЛЕДСТВИЯ И УТОЧНЕНИЯ}

4.1. Явный вид нерезонансной нормальной формы. В классической механике в случае нерезонансных частот имеется явная формула для нормальной формы $H=F\left(p_{1} x_{1}, \ldots, p_{n} x_{n}\right)$ для некоторого формального ряда Тейлора от $n$ переменных с комплексными коэффициентами. Получим подобную формулу для некоммутативных наблюдаемых.

Согласно теореме 2 некоммутативная наблюдаемая приводится к виду $\widehat{H} \in \widehat{\mathbb{N}}_{\lambda}$. В нерезонансном случае $\widehat{\mathbb{N}}_{\lambda}=\bigcup_{\mu \in \mathbb{Z}_{+}^{n}} \mathbb{F}^{\mu, \mu}$. Для упрощения записи обозначим эту алгебру через $\widehat{\mathbb{N}}$.

ПрЕдЛОЖениЕ 9. Пусть $\widehat{F} \in \widehat{\mathbb{N}}$. Тогда существует такой классический формальный рлд Тейлора с комплексными коэффициентами

$$
G\left(y_{0}, \ldots, y_{n}\right)=\sum_{m_{0}, \ldots, m_{n}=0}^{\infty} g_{m_{0}, \ldots, m_{n}} y_{0}^{m_{0}} \ldots y_{n}^{m_{n}}
$$

чmo

$$
\widehat{F}\left(\hat{p}_{1}, \ldots, \hat{p}_{n}, \hat{x}_{1}, \ldots \hat{x}_{n}\right)=G\left(i \mathbf{r}, \hat{I}_{1}, \ldots, \hat{I}_{n}\right),
$$

где $\hat{I}_{1}, \ldots, \hat{I}_{n}$ - следующие взаимно коммутирующие наблюдаемые:

$$
\hat{I}_{j}=\frac{\hat{p}_{j} \circ \hat{x}_{j}+\hat{x}_{j} \circ \hat{p}_{j}}{2} .
$$

Более того, если наблюдаемая $\widehat{F}$ эрмитова, то коэффициенты $g_{m_{0}, \ldots, m_{n}}$ вещественны.

ДокАЗАТЕЛЬСтво. Рассмотрим случай $n=1$. Согласно предложению 2 имеем $\widehat{F}=\Phi(\hat{p} \circ \hat{x}, \hat{x} \circ \hat{p})$, где $\Phi-$ некоторый формальный ряд Тейлора от двух переменных с комплексными коэффициентами. Используя соотношения

$$
\hat{p} \circ \hat{x}=\frac{\hat{I}+\mathbf{r}}{2}, \quad \hat{x} \circ \hat{p}=\frac{\hat{I}-\mathbf{r}}{2},
$$

получаем $\widehat{F}=G(\hat{I}, i \mathbf{r})$ для некоторого формального ряда Тейлора $G$.

Пусть теперь $n>1$. Достаточно доказать предложение для случая, когда $\widehat{F}-$ моном. В этом случае

$$
\widehat{F}=\widehat{F}_{1}\left(\hat{p}_{1}, \hat{x}_{1}\right) \circ \cdots \circ \widehat{F}_{n}\left(\hat{p}_{n}, \hat{x}_{n}\right)
$$

для некоторых мономов $\widehat{F}_{j} \in \mathbb{F}^{\mu_{j}, \mu_{j}}$. Следовательно, для некоторых рядов Тейлора $G_{1}, \ldots, G_{n}$ от двух переменных с комплексными коэффициентами имеем следующее равенство:

$$
\widehat{F}=G_{1}\left(\hat{I}_{1}, i \mathbf{r}\right) \circ \cdots \circ G_{n}\left(\hat{I}_{n}, i \mathbf{r}\right) .
$$

Обозначив правую часть через $G\left(\hat{I}_{1}, \ldots, \hat{I}_{n}, i \mathbf{r}\right)$, получаем для $\widehat{F}$ нужное представление.

Вторая часть утверждения следует из того, что наблюдаемые $i \mathbf{r}$ и $\hat{I}_{j}$ эрмитовы. 
СлЕДСТВИЕ 3. Если $\lambda$ - нерезонансный вектор частот, то для наблюдаемой $\widehat{H}=\widehat{H}_{2}+O_{3}(\hat{p}, \hat{x})$, где $\widehat{H}_{2}$ задано соотношением (11), существует автоморфизм $\widehat{\mathcal{A}} \in \mathrm{Aut} \widehat{\mathbb{H}}$, близкий $\kappa$ тождественному, такой, что

$$
\widehat{\mathcal{A}} \widehat{H}=G\left(\mathbf{r}, \hat{I}_{1}, \ldots, \hat{I}_{n}\right),
$$

где $G$ - формальный ряд Тейлора с комплексными коэффициентами.

\section{2. Эрмитов вид нормальной формы.}

Теорема 3. Пусть $\widehat{H}=\widehat{H}^{*}=\widehat{H}_{2}+O_{3}(\hat{p}, \hat{x})$ - эрмитов гамильтониан с простыми собственными значениями. Тогда:

а) существует автоморфизм $\widehat{\mathcal{A}} \in$ Aut $\widehat{\mathbb{H}}$, близкий $\kappa$ тождественному, такой, что $\left[\widehat{\mathcal{A}} \widehat{H}, \widehat{H}_{2}\right]=0$;

б) если вектор частот $\lambda$ нерезонансный, то существует эрмитов автоморфизм $\widehat{\mathcal{A}} \in \operatorname{Aut} \widehat{\mathbb{H}}($ m.е. переводящий эрмитовы наблюдаемые в эрмитовы) такой, что

$$
\widehat{\mathcal{A}} \widehat{H}=F\left(\widehat{Q}_{1}, \ldots, \widehat{Q}_{n}, i \mathbf{r}\right),
$$

где $F$ - формалъный ряд Тейлора с вещественными коэффициентами от взаимно коммутирующих эрмитовых наблюдаемых $і \mathbf{r}$ и $\widehat{Q}_{1}, \ldots, \widehat{Q}_{n}$ вида

$$
\begin{aligned}
\widehat{Q}_{j} & =\frac{\hat{p}_{j} \circ \hat{x}_{j}+\hat{x}_{j} \circ \hat{p}_{j}}{2}, & j & =1, \ldots, k_{1}, \\
\widehat{Q}_{j} & =\frac{\hat{p}_{j}^{2}+\hat{x}_{j}^{2}}{2}, & j & =k_{1}+1, \ldots, k_{2}, \\
\widehat{Q}_{j} & =\frac{\hat{p}_{j} \circ \hat{x}_{j}+\hat{x}_{j} \circ \hat{p}_{j}}{2}+\frac{\hat{p}_{j+l} \circ \hat{x}_{j+l}+\hat{x}_{j+l} \circ \hat{p}_{j+l}}{2}, & j & =k_{2}+1, \ldots, k_{2}+l, \\
\widehat{Q}_{j+l} & =\hat{p}_{j} \circ \hat{x}_{j+l}-\hat{x}_{j} \circ \hat{p}_{j+l}, & j &
\end{aligned}
$$

где $k_{2}+2 l=n, k_{1} \leqslant k_{2} u k_{1}, k_{2}, l \geqslant 0$.

ДокАЗАтЕЛьСтво. Поскольку $\widehat{H}_{2}$ эрмитова, то классический гамильтониан $H_{2}=$ aver $\widehat{H}_{2}$ является вещественной квадратичной формой. Собственные значения такого гамильтониана $\lambda$ бывают трех типов:

1) вещественная пара: $\lambda= \pm \alpha, \alpha \in \mathbb{R}$;

2) чисто мнимая пара: $\lambda= \pm \beta i, \beta \in \mathbb{R}$;

3) четверка комплексных чисел: $\lambda= \pm \alpha \pm \beta i, \alpha, \beta \in \mathbb{R}$.

Хорошо известно, что существует классический вещественный автоморфизм $\mathcal{L}_{0} \in$ $\mathrm{Aut}_{L} \mathbb{O}$ вида (12), который приводит $H_{2}$ к представлению в виде суммы частичных гамильтонианов от непересекающихся наборов переменных одного из трех типов:

1) $H_{\alpha}=\alpha p x$ для вещественной пары $\lambda= \pm \alpha$;

2) $H_{\beta i}=\beta\left(p^{2}+x^{2}\right) / 2$ для чисто мнимой пары $\lambda= \pm \beta i$;

3) $H_{ \pm \alpha \pm \beta i}=\alpha\left(p_{1} x_{1}+p_{2} x_{2}\right)-\beta\left(p_{1} x_{2}-p_{2} x_{1}\right)$ для комплексной четверки $\lambda= \pm \alpha \pm \beta i$.

По аналогии с теоремой 1 получаем, что существует автоморфизм $\widehat{\mathcal{L}}_{0} \in \mathrm{Aut}_{L} \widehat{\mathbb{H}}$, который приводит $\widehat{H}_{2}$ к виду

$$
\widehat{\mathcal{L}}_{0} \widehat{H}_{2}=\sum_{j=1}^{k_{1}} \alpha_{j} \widehat{Q}_{j}+\sum_{j=k_{1}+1}^{k_{2}} \beta_{j} \widehat{Q}_{j}+\sum_{j=k_{2}+1}^{k_{2}+l}\left(\alpha_{j} \widehat{Q}_{j}+\beta_{j} \widehat{Q}_{j+l}\right)+c i \mathbf{r}, \quad c \in \mathbb{R} .
$$

Автоморфизм $\widehat{\mathcal{L}}_{0}$ эримитов, поскольку $C$ - вещественная матрица. 
Положим $\widehat{\mathcal{H}}=\widehat{\mathcal{L}}_{0} \widehat{H}=\widehat{\mathcal{H}}_{2}+\widehat{\mathcal{H}}_{3}+\cdots$. Если $\widehat{\mathcal{A}} \in \operatorname{Aut} \widehat{\mathbb{H}}-$ эрмитов автоморфизм такой, что $\left[\widehat{\mathcal{A}} \widehat{\mathcal{H}}, \widehat{\mathcal{H}}_{2}\right]=0$, то $\widehat{\mathcal{L}}_{0}^{-1} \widehat{\mathcal{\mathcal { A }}} \widehat{\mathcal{L}}_{0}-$ автоморфизм, требуемый в условии "а". Если собственные значения нерезонансны, а автоморфизм $\widehat{\mathcal{A}} \in$ Aut $\widehat{\mathbb{H}}$ приводит $\widehat{\mathcal{H}}$ к виду (18), то $\widehat{\mathcal{A}} \widehat{\mathcal{L}}_{0}$ - автоморфизм, требуемый в условии "б". Таким образом, не ограничивая общности, считаем, что $\widehat{H}_{2}$ имеет вид (19).

Рассмотрим автоморфизм $\widehat{\mathcal{L}} \in \mathrm{Aut}_{L} \widehat{\mathbb{H}}$, порожденный следующими наблюдаемыми:

$$
\begin{array}{rlrlrl}
\widehat{P}_{j} & =\hat{p}_{j}, & \widehat{X}_{j} & =\hat{x}_{j}, & 1 \leqslant j \leqslant k_{1}, \\
\widehat{P}_{j} & =\frac{\hat{p}_{j}+i \hat{x}_{j}}{\sqrt{2}}, & \widehat{X}_{j} & =\frac{i \hat{p}_{j}+\hat{x}_{j}}{\sqrt{2}}, & & k_{1}+1 \leqslant j \leqslant k_{2}, \\
\widehat{P}_{j} & =\frac{\hat{p}_{j}+\hat{p}_{j+l}}{\sqrt{2}}, & \widehat{X}_{j} & =\frac{\hat{x}_{j}+\hat{x}_{j+l}}{\sqrt{2}}, & & \\
\widehat{P}_{j+l} & =i \frac{\hat{p}_{j+l}-\hat{p}_{j}}{\sqrt{2}}, & \widehat{X}_{j+l} & =i \frac{\hat{x}_{j}-\hat{x}_{j+l}}{\sqrt{2}}, & & k_{2} \leqslant j \leqslant k_{2}+l .
\end{array}
$$

Если $\widehat{H}_{2}$ имеет вид (19), то $\widehat{\mathcal{L}} \widehat{H}_{2}$ имеет вид правой части (11). Воспользуемся теоремой 2 и построим отображение $\widehat{\mathcal{A}} \in$ Aut $\widehat{\mathbb{H}}$, приводящее $\widehat{\mathcal{L}} \widehat{H}$ к нормальной форме. Тогда $\left[\widehat{\mathcal{A}} \widehat{\mathcal{L}} \widehat{H}, \widehat{\mathcal{L}} \widehat{H}_{2}\right]=0$. Следовательно, $\widehat{\mathcal{L}}^{-1} \widehat{\mathcal{A}} \widehat{\mathcal{L}}$ будет требуемым автоморфизмом, если он эрмитов. Кроме того, если собственные значения $\lambda$ нерезонансны, то $\widehat{\mathcal{A}} \widehat{\mathcal{L}} \widehat{H}$ имеет вид правой части (17). Следовательно, если $\widehat{\mathcal{L}}^{-1} \widehat{\mathcal{A}} \widehat{\mathcal{L}}-$ эрмитов, то

$$
\widehat{\mathcal{L}}^{-1} \widehat{\mathcal{A}} \widehat{\mathcal{L}} \widehat{H}=F\left(\widehat{Q}_{1}, \ldots, \widehat{Q}_{n}, i \mathbf{r}\right),
$$

где $\widehat{Q}_{j}$ - наблюдаемые, которые требуются в условии. Таким образом, если мы установим, что $\widehat{\mathcal{L}}^{-1} \widehat{\mathcal{A}} \widehat{\mathcal{L}}$ является эрмитовым автоморфизмом, то теорема будет доказана.

Нормализующее отображение определено в теореме 2 как предел отображений

$$
\widehat{\mathbb{H}} \stackrel{\widehat{\mathcal{A}}^{(1)}}{\longrightarrow} \widehat{\mathbb{H}} \stackrel{\widehat{\mathcal{A}}^{(2)}}{\longrightarrow} \cdots \stackrel{\widehat{\mathcal{A}}^{(m-1)}}{\longrightarrow} \widehat{\mathbb{H}} \stackrel{\widehat{\mathcal{A}}^{(m)}}{\longrightarrow} \cdots,
$$

где $\widehat{\mathcal{A}}^{(m)}=e^{\operatorname{ad}_{\widehat{W}_{m+2}}}$. Введем обозначение $\widehat{\mathcal{A}}_{\mathbb{R}}^{(m)}=e^{\operatorname{ad}_{\widehat{\mathcal{L}}^{-1} \widehat{W}_{m+2}}}$. Диаграмма

$$
\begin{array}{ll}
\widehat{\mathbb{H}} \stackrel{\widehat{\mathcal{A}}_{\mathbb{R}}^{(m)}}{\longrightarrow} \widehat{\mathbb{H}} \\
\downarrow \widehat{\mathcal{L}} \quad & \downarrow \widehat{\mathcal{L}} \\
\widehat{\mathbb{H}} \stackrel{\widehat{\mathcal{A}}^{(m)}}{\longrightarrow} \widehat{\mathbb{H}}
\end{array}
$$

коммутативна. Действительно,

$$
\widehat{\mathcal{L}} \exp \left(\operatorname{ad}_{\widehat{\mathcal{L}}^{-1} \widehat{W}_{m+2}}\right) \widehat{\mathcal{L}}^{-1} \widehat{F}=\widehat{\mathcal{L}} \sum_{j=0}^{\infty} \widehat{\mathcal{L}}^{-1} \frac{\operatorname{ad}_{\widehat{W}_{m+2}^{j}} \widehat{F}}{j !}=\exp \left(\operatorname{ad}_{\widehat{W}_{m+2}}\right) \widehat{F} .
$$

Таким образом, коммутативна и следующая диаграмма:

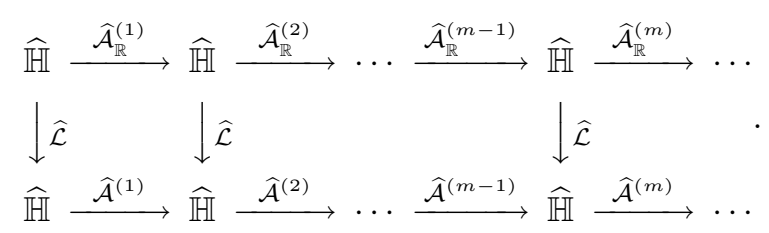


В этой диаграмме предел отображений в верхней цепочке дает автоморфизм $\widehat{\mathcal{L}}^{-1} \widehat{\mathcal{A}} \widehat{\mathcal{L}}$. Нам достаточно доказать, что $\widehat{\mathcal{A}}_{\mathbb{R}}^{(m)}-$ эрмитовы автоморфизмы или что $\widehat{\mathcal{L}}^{-1} \widehat{W}_{m+2}-$ эрмитовы наблюдаемые.

Рассмотрим отображение $\widehat{\mathcal{L}}^{*}$, действующее следующим образом:

$$
\widehat{\mathcal{L}}^{*} \widehat{F}=\widehat{\mathcal{L}} \widehat{F}^{*}, \quad \widehat{F} \in \widehat{\mathbb{H}} .
$$

Это отображение не является автоморфизмом, потому что не выполнено свойство линейности. Тогда условие эрмитовости наблюдаемой $\widehat{\mathcal{L}}^{-1} \widehat{W}_{m+2}$ можно переписать в виде

$$
\widehat{\mathcal{L}}\left(\widehat{\mathcal{L}}^{-1}\right)^{*} \widehat{W}_{m+2}^{*}=\widehat{W}_{m+2} .
$$

Обозначим $\widehat{\mathcal{L}}^{\prime}=\widehat{\mathcal{L}}\left(\widehat{\mathcal{L}}^{-1}\right)^{*}$. Отображение $\widehat{\mathcal{L}}^{\prime}$ является автоморфизмом ассоциативной алгебры и алгебры Ли $\widehat{\mathbb{H}}$, который порождается наблюдаемыми

$$
\begin{aligned}
& \widehat{P}_{j}=\hat{p}_{j}, \quad \widehat{X}_{j}=\hat{x}_{j}, \\
& \widehat{P}_{j}=i \hat{x}_{j}, \quad \widehat{X}_{j}=i \hat{p}_{j}, \\
& j=1, \ldots, k_{1}, \\
& \widehat{P}_{j}=\hat{p}_{j+l}, \quad \widehat{X}_{j}=\hat{x}_{j+l}, \quad \widehat{P}_{j+l}=\hat{p}_{j}, \quad \widehat{X}_{j+l}=\hat{x}_{j}, \quad j=k_{2}, \ldots, k_{2}+l .
\end{aligned}
$$

Итак, докажем эрмитовость наблюдаемых $\widehat{\mathcal{L}}^{-1} \widehat{W}_{m}$ по индукции. Пусть наблюдаемые $\widehat{\mathcal{L}}^{-1} \widehat{W}_{3}, \ldots, \widehat{\mathcal{L}}^{-1} \widehat{W}_{m+1}$ эрмитовы. Тогда наблюдаемая $\widehat{\mathcal{A}}_{\mathbb{R}}^{(m-1)} \ldots \widehat{\mathcal{A}}_{\mathbb{R}}^{(1)} \widehat{H}$ также эрмитова. Обозначим

$$
\widehat{\mathcal{H}}=\widehat{\mathcal{L}} \widehat{\mathcal{A}}_{\mathbb{R}}^{(m-1)} \ldots \widehat{\mathcal{A}}_{\mathbb{R}}^{(1)} \widehat{H}=\widehat{\mathcal{H}}_{2}+\widehat{\mathcal{H}}_{3}+\cdots, \quad \widehat{\mathcal{H}}_{k} \in \mathbb{F}_{k},
$$

где $\widehat{\mathcal{H}}_{2}$ имеет вид правой части $(11)$. Тогда $\widehat{W}_{m+2}$ определяется из условия

$$
\widehat{\mathcal{H}}_{m+2}+\left[\widehat{W}_{m+2}, \widehat{\mathcal{H}}_{2}\right] \in \widehat{\mathbb{N}}_{\lambda}, \quad \widehat{\mathcal{L}}^{\prime} \widehat{\mathcal{H}}_{m+2}^{*}=\widehat{\mathcal{H}}_{m+2}, \quad \widehat{\mathcal{L}}^{\prime} \widehat{\mathcal{H}}_{2}^{*}=\widehat{\mathcal{H}}_{2} .
$$

Докажем, что выполнено соотношение (20). Пусть $\widehat{\mathcal{H}}_{m+2}=\widehat{F}_{0}+\widehat{F}_{1}$, где $\widehat{F}_{0} \in \mathbb{N}_{\lambda}$ и $\widehat{F}_{1} \in \mathbb{N}_{\lambda}^{\mathrm{c}}$. Тогда $(21)$ записывается в виде

$$
\widehat{F}_{1}+\left[\widehat{W}_{m+2}, \widehat{\mathcal{H}}_{2}\right]=0, \quad \widehat{W}_{m+2} \in \widehat{\mathbb{N}}_{\lambda}^{c} .
$$

Заметим, что пространства $\mathbb{N}_{\lambda}$ и $\mathbb{N}_{\lambda}^{c}$ являются инвариантными относительно отображения $\widehat{\mathcal{L}}^{\prime}$, поэтому $\widehat{\mathcal{L}}^{\prime} \widehat{F}_{1}^{*}=\widehat{F}_{1}$. Следовательно,

$$
\widehat{F}_{1}+\left[\widehat{\mathcal{L}}^{\prime} \widehat{W}_{m+2}^{*}, \widehat{\mathcal{H}}_{2}\right]=\widehat{\mathcal{L}}^{\prime} \widehat{F}_{1}^{*}+\left[\widehat{\mathcal{L}}^{\prime} \widehat{W}_{m+2}^{*}, \widehat{\mathcal{L}}^{\prime} \widehat{\mathcal{H}}_{2}^{*}\right]=0 .
$$

Согласно следствию 2 получаем $\widehat{W}_{m+2}=\widehat{\mathcal{L}}^{\prime} \widehat{W}_{m+2}^{*}$, что и требовалось доказать.

\section{3. Вопросы единственности.}

Теорема 4. Пусть $\widehat{\mathcal{A}} \in$ Aut $\widehat{\mathbb{H}}$ - автоморфизм, близкий $к$ тождественному. Тогда следующие три утверждения эквивалентны:

1) существует наблюдаемая $\widehat{H} \in \widehat{\mathbb{N}}_{\lambda}$ такая, что $\widehat{H}=\widehat{H}_{2}+O_{3}(\hat{p}, \hat{x})$, где $\widehat{H}_{2}$ имеет вид правой части (11), и, кроме того, $\widehat{\mathcal{A}} \widehat{H} \in \widehat{\mathbb{N}}_{\lambda}$;

2) $\widehat{\mathcal{A}}\left(\widehat{\mathbb{N}}_{\lambda}\right)=\widehat{\mathbb{N}}_{\lambda}$;

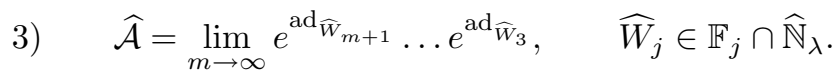


ДоКАЗАТЕЛЬСтво. $2 \Longrightarrow 1$. Эта импликация тривиальна.

$3 \Longrightarrow 2$. Поскольку $\widehat{\mathbb{N}}_{\lambda}$ является подалгеброй Ли $\widehat{\mathbb{H}}$, то для всякой наблюдаемой $\widehat{H} \in \widehat{\mathbb{N}}_{\lambda}$ выполнено $e^{\operatorname{ad}_{\widehat{w}_{j}}} \widehat{H} \in \widehat{\mathbb{N}}_{\lambda}$. Следовательно, $\widehat{\mathcal{A}}\left(\widehat{\mathbb{N}}_{\lambda}\right) \subset \widehat{\mathbb{N}}_{\lambda}$, что равносильно условию 2 , поскольку $\widehat{\mathcal{A}}$ - изоморфизм $\widehat{\mathbb{H}}$.

$1 \Longrightarrow 3$. Согласно предложению 4 о приближении автоморфизма выполнено (10). Докажем, что $\widehat{W}_{j} \in \widehat{\mathbb{N}}_{\lambda}$.

Пусть $\widehat{H}=\widehat{H}_{2}+\widehat{H}_{3}+\cdots$, где $\widehat{H}_{2}$ задается формулой (11). Тогда

$$
\widehat{\mathcal{A}} \widehat{H}=\widehat{H}_{2}+\widehat{H}_{3}+\left[\widehat{W}_{3}, \widehat{H}_{2}\right]+O_{4}(\hat{p}, \hat{x}) .
$$

Поскольку $\widehat{H}_{3} \in \widehat{\mathbb{N}}_{\lambda}$, должно быть выполнено условие $\left[\widehat{W}_{3}, \widehat{H}_{2}\right] \in \widehat{\mathbb{N}}_{\lambda}$. Это условие равносильно тому, что $\widehat{W}_{3} \in \widehat{\mathbb{N}}_{\lambda}$.

Обозначим $e^{\operatorname{ad}_{\widehat{W}_{3}} \widehat{H}}$ через $\widehat{H}$, a $\lim _{m \rightarrow \infty} e^{\operatorname{ad}_{\widehat{W}_{m+1}}} \ldots e^{\operatorname{ad}_{\widehat{W}_{4}}}$ через $\widehat{\mathcal{A}}$ и проведем те же самые рассуждения. Получаем $\widehat{W}_{4} \in \widehat{\mathbb{N}}_{\lambda}$. Продолжая эту процедуру, получаем доказательство нашей импликации. Теорема доказана.

СлеДСтвиЕ 4. Пусть $\widehat{H}=\widehat{H}_{2}+O_{3}(\hat{p}, \hat{x})$, где $\widehat{H}_{2}$ задается в (11). Пусть $\widehat{\mathcal{B}}_{1}, \widehat{\mathcal{B}}_{2} \in$ Aut $\widehat{\mathbb{H}}$ - близкие $\kappa$ тождественному нормализующие автоморфизмы, т.е. такие, что $\widehat{\mathcal{B}}_{1} \widehat{H}, \widehat{\mathcal{B}}_{2} \widehat{H} \in \widehat{\mathbb{N}}_{\lambda}$. Тогда $\widehat{\mathcal{B}}_{1}=\widehat{\mathcal{A}} \widehat{\mathcal{B}}_{2}$, где $\widehat{\mathcal{A}} \in$ Aut $\widehat{\mathbb{H}}$ имеет вид (22).

Если вектор частот $\lambda$ нерезонансныи, то $\widehat{\mathcal{B}}_{1} \widehat{H}=\widehat{\mathcal{B}}_{2} \widehat{H}, u$

$$
\widehat{\mathcal{A}}=e^{\operatorname{ad}_{\widehat{W}}}, \quad \widehat{W}=O_{3}(\hat{p}, \hat{x}), \quad \widehat{W} \in \widehat{\mathbb{N}}_{\lambda} .
$$

ДокАЗАтЕЛЬство. Это утверждение получается применением теоремы 4 для $\widehat{\mathcal{B}}_{2} \widehat{\mathcal{B}}_{1}^{-1}$. Если $\lambda$ - нерезонансный вектор, то $\widehat{\mathbb{N}}_{\lambda}-$ коммутативная подалгебра. Следовательно, автоморфизм, определенный формулой (22), действует тождественно на $\widehat{\mathbb{N}}_{\lambda}$. Представление $(23)$ следует из того, что $e^{\operatorname{ad}_{\widehat{U}}} e^{\operatorname{ad}_{\widehat{V}}}=e^{\operatorname{ad}_{\widehat{U}+\hat{V}}}$, если $[\widehat{U}, \widehat{V}]=0$.

Итак, преобразование, нормализующее данную наблюдаемую, единственно с точностью до композиции с отображением вида (22). Сам вид нормальной формы единствен только в нерезонансном случае. Если вектор частот резонансный, то нормальная форма определена с точностью до применения отображения (22).

\section{Доказательство предложения 3}

ПРИЛОЖЕНИЕ

Идею этого доказательства автору сообщил Д. В. Трещев. Заметим, что нижний индекс наблюдаемых здесь вовсе не обозначает степень однородной формы.

Ясно, что существует наблюдаемая $\widehat{W}_{1} \in \widehat{\mathbb{H}}$ такая, что $\operatorname{ad}_{\hat{p}_{1}} \widehat{W}_{1}=-\widehat{P}_{1}$. Положим $\widehat{P}_{j}^{(1)}=\widehat{P}_{j}+\operatorname{ad}_{\hat{p}_{j}} \widehat{W}_{1}, \widehat{X}_{j}^{(1)}=\widehat{X}_{j}+\operatorname{ad}_{\hat{x}_{j}} \widehat{W}_{1}$. Тогда $\widehat{P}_{1}^{(1)}=0$. Кроме того,

$$
\operatorname{ad}_{\hat{p}_{1}} \widehat{P}_{j}^{(1)}=\operatorname{ad}_{\hat{p}_{1}} \widehat{P}_{j}+\operatorname{ad}_{\hat{p}_{1}} \operatorname{ad}_{\hat{p}_{j}} \widehat{W}_{1}=\operatorname{ad}_{\hat{p}_{1}} \widehat{P}_{j}+\operatorname{ad}_{\hat{p}_{j}} \operatorname{ad}_{\hat{p}_{1}} \widehat{W}_{1}=\operatorname{ad}_{\hat{p}_{1}} \widehat{P}_{j}-\operatorname{ad}_{\hat{p}_{j}} \widehat{P}_{1}=0 .
$$

В последнем равенстве мы воспользовались условием (9). Аналогично, используя (9), а также равенство $\operatorname{ad}_{\hat{p}_{j}} \operatorname{ad}_{\hat{x}_{k}}=\operatorname{ad}_{\hat{x}_{k}} \operatorname{ad}_{\hat{p}_{j}}$, мы получаем $\operatorname{ad}_{\hat{p}_{1}} \widehat{X}_{j}^{(1)}=0$. Говоря неформально, это означает, что зависимость $\widehat{P}_{j}^{(1)}$ и $\widehat{X}_{j}^{(1)}$ от $\hat{x}_{1}$ есть зависимость только от $\mathbf{r}=\hat{p}_{1} \circ \hat{x}_{1}-\hat{x}_{1} \circ \hat{p}_{1}$. 
Далее, возьмем такую наблюдаемую $\widehat{W}_{2}$, что выполнено соотношение

$$
\operatorname{ad}_{\hat{p}_{2}} \widehat{W}_{2}=-\widehat{P}_{2}^{(1)}
$$

и положим

$$
\widehat{P}_{j}^{(2)}=\widehat{P}_{j}^{(1)}+\operatorname{ad}_{\hat{p}_{j}} \widehat{W}_{2}, \quad \widehat{X}_{j}^{(2)}=\widehat{X}_{j}^{(1)}+\operatorname{ad}_{\hat{x}_{j}} \widehat{W}_{2} .
$$

Тогда

$$
\operatorname{ad}_{\hat{p}_{2}} \widehat{P}_{j}^{(2)}=\operatorname{ad}_{\hat{p}_{2}} \widehat{P}_{j}^{(1)}+\operatorname{ad}_{\hat{p}_{2}} \operatorname{ad}_{\hat{p}_{j}} \widehat{W}_{2}=\operatorname{ad}_{\hat{p}_{2}} \widehat{P}_{j}^{(1)}-\operatorname{ad}_{\hat{p}_{j}} \widehat{P}_{2}^{(1)}=\operatorname{ad}_{\hat{p}_{2}} \widehat{P}_{j}-\operatorname{ad}_{\hat{p}_{j}} \widehat{P}_{2}=0 .
$$

Аналогично, $\operatorname{ad}_{\hat{p}_{2}} \widehat{X}_{j}^{(2)}=0$.

Теперь покажем, что

$$
\operatorname{ad}_{\hat{p}_{1}} \widehat{P}_{j}^{(2)}=\operatorname{ad}_{\hat{p}_{1}} \widehat{X}_{j}^{(2)}=0
$$

Действительно,

$$
\operatorname{ad}_{\hat{p}_{1}} \widehat{P} j^{(2)}=\operatorname{ad}_{\hat{p}_{1}} \widehat{P}_{j}^{(1)}+\operatorname{ad}_{\hat{p}_{1}} \operatorname{ad}_{\hat{p}_{j}} \widehat{W}_{2}=\operatorname{ad}_{\hat{p}_{j}} \operatorname{ad}_{\hat{p}_{1}} \widehat{W}_{2} \text {. }
$$

Аналогично, $\operatorname{ad}_{\hat{p}_{1}} \widehat{X}_{j}^{(2)}=\operatorname{ad}_{\hat{x}_{j}} \operatorname{ad}_{\hat{p}_{1}} \widehat{W}_{2}$. Для того чтобы выполнялось равенство (25), достаточно выбрать $\widehat{W}_{2}$, удовлетворяющее $(24)$, так, чтобы $\operatorname{ad}_{\hat{p}_{1}} \widehat{W}_{2}=0$. Иначе говоря, пользуясь тем, что в формуле $(24) \widehat{P}_{1}^{(2)}$ не зависит от $\hat{x}_{1}$, мы выбираем $\widehat{W}_{2}$ также не зависящим от $\hat{x}_{1}$.

Будем использовать разложение наблюдаемых по степеням $\mathbf{r}$. Пусть $\widehat{W}_{2}=$ $\sum C_{l, \mu, \nu} \mathbf{r}^{l} \circ \hat{p}^{\mu} \circ \hat{x}^{\nu}$. Ясно, что $\operatorname{ad}_{\hat{p}_{j}} \widehat{W}=0$ тогда и только тогда, когда $C_{l, \mu, \nu}=0$ для $\nu_{j} \neq 0$. Положим $\widehat{W}_{2}^{\prime}=\sum_{\nu_{2} \neq 0} C_{l, \mu, \nu} \mathbf{r}^{l} \circ \hat{p}^{\mu} \circ \hat{x}^{\nu}$. Тогда

$$
-\widehat{P}_{2}^{(1)}=\operatorname{ad}_{\hat{p}_{2}} \widehat{W}_{2}=\operatorname{ad}_{\hat{p}_{2}} \widehat{W}_{2}^{\prime}=\sum \nu_{2} C_{l, \mu, \nu} \mathbf{r}^{l} \circ \hat{p}^{\mu} \circ \hat{x}^{\nu}
$$

Поскольку $\operatorname{ad}_{\hat{p}_{1}} \widehat{P}_{2}^{(1)}=0$, то $C_{l, \mu, \nu}=0$, если $\nu_{1} \neq 0$. Следовательно, $\operatorname{ad}_{\hat{p}_{1}} \widehat{W}_{2}^{\prime}=0$.

Для упрощения записи наблюдаемую $\widehat{W}_{2}^{\prime}$ будем обозначать по-прежнему через $\widehat{W}_{2}$. Тогда $\operatorname{ad}_{\hat{p}_{1}} \widehat{P}_{j}^{(2)}=\operatorname{ad}_{\hat{p}_{2}} \widehat{P}_{j}^{(2)}=\operatorname{ad}_{\hat{p}_{1}} \widehat{X}_{j}^{(2)}=\operatorname{ad}_{\hat{p}_{2}} \widehat{X}_{j}^{(2)}=0$.

Аналогичным образом строим $\widehat{P}_{j}^{(k)}, \widehat{X}_{j}^{(k)}, k=1, \ldots, n$, так, чтобы

$$
\begin{aligned}
\operatorname{ad}_{\hat{p}_{1}} \widehat{P}_{j}^{(k)} & =\operatorname{ad}_{\hat{p}_{2}} \widehat{P}_{j}^{(k)}=\cdots=\operatorname{ad}_{\hat{p}_{k}} \widehat{P}_{j}^{(k)}=0, \\
\operatorname{ad}_{\hat{p}_{1}} \widehat{X}_{j}^{(k)} & =\operatorname{ad}_{\hat{p}_{2}} \widehat{X}_{j}^{(k)}=\cdots=\operatorname{ad}_{\hat{p}_{k}} \widehat{X}_{j}^{(k)}=0 .
\end{aligned}
$$

Затем построим $\widehat{P}_{j}^{(k)}, \widehat{X}_{j}^{(k)}, k=n+1, \ldots, 2 n$, так, чтобы

$$
\begin{aligned}
& \operatorname{ad}_{\hat{p}_{l}} \widehat{P}_{j}^{(k)}=\operatorname{ad}_{\hat{p}_{l}} \widehat{X}_{j}^{(k)}=0, \quad l=1, \ldots, n, \\
& \operatorname{ad}_{\hat{x}_{1}} \widehat{P}_{j}^{(k)}=\operatorname{ad}_{\hat{x}_{2}} \widehat{P}_{j}^{(k)}=\cdots=\operatorname{ad}_{\hat{x}_{k-n}} \widehat{P}_{j}^{(k)}=0, \\
& \operatorname{ad}_{\hat{x}_{1}} \widehat{X}_{j}^{(k)}=\operatorname{ad}_{\hat{x}_{2}} \widehat{X}_{j}^{(k)}=\cdots=\operatorname{ad}_{\hat{x}_{k}} \widehat{X}_{j}^{(k)}=0 .
\end{aligned}
$$

В итоге получаем

$$
\widehat{P}_{j}^{(2 n)}=\widehat{P}_{j}+\operatorname{ad}_{\hat{p}_{j}} \widehat{W}, \quad \widehat{X}_{j}^{(2 n)}=\widehat{X}_{j}+\operatorname{ad}_{\hat{x}_{j}} \widehat{W},
$$


где $\widehat{W}=\widehat{W}_{1}+\cdots+\widehat{W}_{2 n}$, причем

$$
\operatorname{ad}_{\hat{p}_{k}} \widehat{P}_{j}^{(2 n)}=a d_{\hat{x}_{k}} \widehat{P}_{j}^{(2 n)}=\operatorname{ad}_{\hat{p}_{k}} \widehat{G}_{j}^{(2 n)}=\operatorname{ad}_{\hat{x}_{k}} \widehat{X}_{j}^{(2 n)}=0 .
$$

Таким образом,

$$
\widehat{P}_{j}-\operatorname{ad}_{\widehat{W}} \hat{p}_{j}=\hat{\xi}_{j}, \quad \widehat{X}_{j}-\operatorname{ad}_{\widehat{W}} \hat{x}_{j}=\hat{\eta}_{j}, \quad \hat{\xi}_{j}, \hat{\eta}_{k} \in \hat{J}_{0} .
$$

Положив $\widehat{W}_{0}=\sum_{j=1}^{n} \hat{x}_{j} \circ \hat{\xi}_{j}-\sum_{j=1}^{n} \hat{p}_{j} \circ \hat{\eta}_{j}$, получаем

$$
\widehat{P}_{j}=\operatorname{ad}_{\widehat{W}-\widehat{W}_{0}} \hat{p}_{j}, \quad \widehat{X}_{j}=\operatorname{ad}_{\widehat{W}-\widehat{W}_{0}} \hat{x}_{j}
$$

Предложение доказано.

Благодарности. Автор выражает благодарность Д. В. Трещеву за полезные дискуссии, внимание к работе и идею доказательства предложения 3. Работа была выполнена при поддержке РФФИ (грант № 08-01-00681) и Программы поддержки ведущих научных школ (грант НШ-691.2008.1).

\section{Список литературы}

[1] Дж. Д. Биркгоф, Динамические системы, Гостехиздат, М., 1941.

[2] T. M. Cherry, Proc. London Math. Soc., 27:2 (1927), 151-170.

[3] J. Moser, Comm. Pure Appl. Math., 9:4 (1956), 673-692.

[4] А. Д. Брюно, Локальный метод нелинейного анализа дифференииальных уравнений, Наука, М., 1979.

[5] Д. Трещёв, Тр. МИАН, 250 (2005), 226-261.

[6] Д. В. Трещёв, Тр. МИАН, 259 (2007), 203-242.

[7] Ю. В. Егоров, УМH, 24:5(149) (1969), 235-236.

[8] J. J. Duistermaat, L. Hörmander, Acta Math., 128:1 (1972), 183-269.

[9] L. Hörmander, Acta Math., 127:1 (1971), 79-183.

[10] J. Bellissard, M. Vittot, Ann. Inst. H. Poincaré Phys. Théor., 52:3 (1990), 175-235.

[11] J. Sjöstrand, Asymptotic Anal., 6:1 (1992), 29-43.

[12] C. Gérard, J. Sjöstrand, Comm. Math. Phys., 108:3 (1987), 391-421.

[13] J. Sjöstrand, Asymptotic Anal., 36:2 (2003), 93-113.

[14] N. Kaidi, P. Kerdelhuré, Asymptot. Anal., 23:1 (2000), 1-21.

[15] A. Iantchenko, J. Sjöstrand, Amer. J. Math., 124:4 (2002), 817-850.

[16] A. Anikin, Regul. Chaotic Dyn., 13:5 (2008), 377-402.

[17] M. Karasev, "Noncommutative algebras, nano-structures, and quantum dynamics generated by resonances", Quantum Algebras and Poisson Geometry in Mathematical Physics, AMS Transl. Ser. 2, 216, ed. M. V. Karasev, AMS, Providence, RI, 2005, 1-17; Adv. Stud. Contemp. Math., 11 (2005), 33-56; Russ. J. Math. Phys., 13:2 (2006), 131-150.

[18] М. В. Карасев, В. П. Маслов, УМН, 39:6(240) (1984), 115-173. 